\title{
AFTER THE INTERACTION: AN EFFICIENTLY STAR-FORMING MOLECULAR DISK IN NGC 5195
}

\author{
Katherine Alatalo ${ }^{1,8}$, Rebeca Aladro ${ }^{2,3}$, Kristina Nyland ${ }^{4}$, Susanne Aalto ${ }^{3}$, Theodoros Bitsakis ${ }^{5}$, \\ JOHN S. Gallagher ${ }^{6}$, AND LaURANne LANZ ${ }^{7}$ \\ ${ }^{1}$ Observatories of the Carnegie Institution of Washington, 813 Santa Barbara Street, Pasadena, CA 91101, USA; kalatalo@carnegiescience.edu \\ ${ }^{2}$ European Southern Observatory, Avda. Alonso de Córdova 3107, Vitacura, Santiago, Chile \\ ${ }^{3}$ Department of Earth and Space Sciences, Chalmers University of Technology, Onsala Observatory, 43994 Onsala, Sweden \\ ${ }^{4}$ National Radio Astronomy Observatory, 520 Edgemont Road, Charlottesville, VA 22903, USA \\ ${ }^{5}$ Instituto de Radioastronomía y Astrofísica, Universidad Nacional Autónoma de México, C.P. 58190, Morelia, Mexico \\ ${ }^{6}$ Department of Astronomy, University of Wisconsin-Madison, 5534 Sterling, 475 North Charter Street, Madison WI 53706, USA \\ ${ }^{7}$ Infrared Processing and Analysis Center, California Institute of Technology, MC100-22, Pasadena, CA 91125, USA \\ Received 2016 April 27; revised 2016 July 22; accepted 2016 August 3; published 2016 October 18
}

\begin{abstract}
We present new molecular gas maps of NGC 5195 (alternatively known as M51b) from the Combined Array for Research in Millimeter Astronomy, including ${ }^{12} \mathrm{CO}(1-0),{ }^{13} \mathrm{CO}(1-0), \mathrm{CN}\left(1_{0,2}-0_{0,1}\right), \mathrm{CS}(2-1)$, and $3 \mathrm{~mm}$ continuum. We also detected $\mathrm{HCN}(1-0)$ and $\mathrm{HCO}^{+}(1-0)$ using the Onsala Space Observatory. NGC 5195 has a ${ }^{12} \mathrm{CO} /{ }^{13} \mathrm{CO}$ ratio $\left(\mathcal{R}_{12 / 13}=11.4 \pm 0.5\right)$ consistent with normal star-forming galaxies. The $\mathrm{CN}(1-0)$ intensity is higher than is seen in an average star-forming galaxy, possibly enhanced in the diffuse gas in photo-dissociation regions. Stellar template fitting of the nuclear spectrum of NGC 5195 shows two stellar populations: an 80\% mass fraction of old $(\gtrsim 10 \mathrm{Gyr})$ and a $20 \%$ mass fraction of intermediate-aged $(\approx 1 \mathrm{Gyr})$ stellar populations. This provides a constraint on the timescale over which NGC 5195 experienced enhanced star formation during its interaction with M51a. The average molecular gas depletion timescale in NGC 5195 is $\left\langle\tau_{\text {dep }}\right\rangle=3.08$ Gyr, a factor of $\approx 2$ larger than the depletion timescales in nearby star-forming galaxies, but consistent with the depletion seen in CO-detected early-type galaxies. While radio continuum emission at centimeter and millimeter wavelengths is present in the vicinity of the nucleus of NGC 5195, we find it is most likely associated with nuclear star formation rather than radio-loud AGN activity. Thus, despite having a substantial interaction with M51a 1/2 Gyr ago, the molecular gas in NGC 5195 has resettled and is currently forming stars at an efficiency consistent with settled early-type galaxies.
\end{abstract}

Key words: galaxies: elliptical and lenticular, cD - galaxies: individual (NGC 5195) - galaxies: interactions galaxies: ISM - radio lines: galaxies

\section{INTRODUCTION}

Galaxy populations in the present-day universe largely populate a bimodal distribution between blue and red optical colors with few objects occupying an intermediate color "green valley" (Baade 1958; Holmberg 1958; Tinsley 1978; Strateva et al. 2001; Baldry et al. 2004). Slowly evolving galaxies would not necessarily pass rapidly through a phase with intermediate colors. Thus, the slow overall evolution of galaxies since $z \sim 1$ in combination with the increasing fraction of galaxies on the "red sequence" suggests that galaxies in the green valley experienced a rapid truncation or quenching of their star formation (Bell et al. 2003; Faber et al. 2007). However, the existence of spirals with intermediate colors that appear to be evolving at secular rates suggests the existence of an alternate path to the green valley. The intermediate colors in these spirals could result from the steady build-up long-lived lower mass stars leading to a more leisurely transition through the green valley. Therefore, two types of galaxies with intermediate colors may exist. Those produced by slow evolution and rapid quenching events, that cannot be separated on the basis of broadband optical colors alone.

Other types of observations, however, can be used to separate these two types of green valley galaxies. Systems where star-formation rates (SFRs) have rapidly changed show distinct signposts, including unusual infrared colors. Johnson et al. (2007) and Walker et al. (2010) were able to show that the

\footnotetext{
${ }^{8}$ Hubble Fellow.
}

Spitzer colors of Hickson Compact Group galaxies (a known rapidly transitioning population; Hickson 1982) showed a dearth of intermediate Spitzer Infrared Array Camera (IRAC) colors (Lacy et al. 2004), suggestive of rapid transformation. While the galaxy population as a whole did not show bimodality in Spitzer colors, it did strongly bifurcate using WISE [4.6]-[12] $\mu \mathrm{m}$ colors (Ko et al. 2013; Alatalo et al. 2014; Yesuf et al. 2014). In the modern universe $(z \sim 0)$, this transformation appears to be permanent (Appleton et al. 2014; Young et al. 2014); thus understanding all pathways that can lead a blue late-type galaxy to become a red early type is essential.

Until recently, it was assumed that in order for a galaxy to metamorphose, it would have to shed its interstellar medium as it quenched star formation (Hopkins et al. 2006), but evidence is mounting that this is not strictly necessary. Through the ATLAS $^{3 \mathrm{D}}$ survey, Young et al. (2011) showed that at least $22 \%$ of early-type galaxies contain a molecular reservoir, although for the most part the molecular gas fraction of these systems did not exceed 1\% (save for the extraordinary galaxy NGC 1266; Alatalo et al. 2011). French et al. (2015) and Rowlands et al. (2015) showed that there were also substantial molecular reservoirs in poststarburst galaxies, a subset of objects known to have rapidly quenched their star formation (Dressler \& Gunn 1983; Zabludoff et al. 1996; Quintero et al. 2004). Shocked poststarburst galaxies (Alatalo et al. 2016a), identified based on evidence of intermediateaged stars and a lack of star formation from their ionized gas 
Table 1

Properties of NGC 5195

\begin{tabular}{lll}
\hline \hline R.A. (J2000) & $13^{\mathrm{h}} 29^{\mathrm{m}} 59^{\mathrm{s}} .59$ & $(1)$ \\
Decl. (J2000) & $+47^{\circ} 15^{\mathrm{m}} 58^{\mathrm{s}} .1$ & $(1)$ \\
Morphology & $\mathrm{I} 0$ Peculiar & $(2)$ \\
Distance & $9.9 \mathrm{Mpc}$ & $(3)$ \\
Position Angle & $+101^{\circ}(\mathrm{CCW}$ from $\mathrm{N})$ & $(2)$ \\
Inclination Angle & $37^{\circ}\left(\right.$ face-on $\left.=0^{\circ}\right)$ & $(2)$ \\
Nuclear activity & AGN $/$ LINER & $(4)$ \\
$\log \left(L_{\mathrm{FIR}}\right)$ & $9.3 L_{\odot}$ & $(5)$ \\
$M_{\star}$ & $1.95 \times 10^{10} M_{\odot}$ & $(6)$
\end{tabular}

Note. (1) Skrutskie et al. (2006); (2) de Vaucouleurs et al. (1991); (3) Tikhonov et al. (2009); (4) Moustakas et al. (2010); (5) Dale et al. (2009); (6) Lanz et al. (2013).

ratios also contain substantial gas reservoirs (Alatalo et al. 2016b), despite it being suspected that their metamorphosis started from blue, star-forming spiral to red, quiescent early-type galaxies.

Alatalo et al. (2015a) surveyed the molecular gas content in transitioning Hickson Compact Group galaxies (Cluver et al. 2013; Lisenfeld et al. 2014) using the Combined Array for Research in Millimeter Astronomy (CARMA; Bock et al. 2006) ${ }^{9}$ and found that the molecular gas fractions in this subset of transitioning galaxies are not related to their color (and thus, the transition phase), but rather the star-formation efficiency is related to the color. Alatalo et al. (2015a) suggest that the inability for the molecular gas to form stars efficiently has more influence on a galaxy's path onto the red sequence than the total content of molecular material available. Studies by the ATLAS ${ }^{3 \mathrm{D}}$ team seem to support this, with early-type galaxies showing slightly suppressed star formation as well (Davis et al. 2014), although a direct color comparison has yet to be done on these sources.

NGC 5195 (alternatively known as M51b) is the disky barred (SB0/SBa(s)) galaxy (Spillar et al. 1992) that is the low-mass companion of NGC 5194 (alternatively known as M51a or "the Whirlpool Galaxy"), having undergone a 3:1 interaction roughly 340-500 Myr ago (Salo \& Laurikainen 2000; Dobbs et al. 2010; Mentuch Cooper et al. 2012). NGC 5195 is a good case study of how star formation quenches for a galaxy a short time after an interaction, being nearby and showing signs of having recently transitioned. The integrated $u-r$ color $^{10}$ of NGC 5195 is 2.25, which combined with its mass (Table 1), places it on the cusp between the red sequence and the green valley (Schawinski et al. 2014). Centrally concentrated molecular gas (Aalto \& Rydbeck 2001), and bright nuclear infrared emission (Boulade et al. 1996) further support that NGC 5195 is in the process of becoming a poststarburst (Dressler \& Gunn 1983; Zabludoff et al. 1996), and finally an early-type red-sequence galaxy, traversing the "standard" pathway for galaxy transitions (Hopkins et al. 2008).

Table 1 lists the basic properties of NGC 5195 used for this paper. Mid-infrared (IR) spectra of NGC 5195 confirm the presence of polycyclic aromatic hydrocarbon (PAH) emission (Boulade et al. 1996; Roussel et al. 2007), which is more consistent with a poststarburst stellar population than an ongoing starburst. Lanz et al. (2013) used the far-ultraviolet

\footnotetext{
9 http://www.mmarray.org

${ }^{10}$ Using the SDSS DR12 $u$ and $r$ modelmags (Alam et al. 2015).
}

(UV) to the far-IR spectral energy distribution (SED) of NGC 5195 and were able to estimate a modest SFR of $0.142 M_{\odot} \mathrm{yr}^{-1}$. The warm $\mathrm{H}_{2}$ emission detected with the Spitzer Infrared Spectrograph is consistent with that of other star-forming galaxies in the SINGS sample (Roussel et al. 2007). Mentuch Cooper et al. (2012) also fitted the UV to far-IR photometry and concluded that NGC 5195 is a poststarburst. Although Schweizer (1977) detected ionized gas emission lines in the galaxy, Ho et al. (1997) and Moustakas et al. (2010) found that the ionized gas line ratios are consistent with a low ionization nuclear emission line region (Kewley et al. 2006). Weak X-ray emission was found coincident with a radio source, possibly due to an active galactic nucleus (AGN), but also consistent with an ultra-luminous X-ray source origin (Terashima \& Wilson 2004; Schlegel et al. 2016).

Sage (1990) mapped NGC 5195 in ${ }^{12} \mathrm{CO}(1-0)$, and observed ${ }^{12} \mathrm{CO}(2-1)$ and ${ }^{13} \mathrm{CO}(1-0)$ with the National Radio Astronomy Observatory (NRAO) $12 \mathrm{~m}$ single dish, showing that there was molecular gas in the galaxy. Kohno et al. (2002) were able to detect $\mathrm{HCN}(1-0)$ using the Nobeyama Radio Observatory (NRO) $45 \mathrm{~m}$ single dish, and mapped ${ }^{12} \mathrm{CO}(1-0)$ using the Nobeyama Millimeter Array (NMA). Kohno et al. (2002) showed that the molecular gas is concentrated in the center of NGC 5195, and claimed that the molecular gas is too stable to form stars.

We present new molecular gas maps of NGC 5195, and use them to develop a narrative of the star formation from the time of its interaction with M51a. In Section 2, we describe the observations from CARMA, including reduction and analysis methods. In Section 3, we discuss the properties of the molecular gas of NGC 5195. In Section 4 we discuss the past and present star formation in NGC 5195. In Section 5, we present our conclusions. We use the common distance to M51a and NGC 5195 of $9.9 \mathrm{Mpc}$, following Tikhonov et al. (2009) who used the Hertzsprung-Russell diagram of individual resolved stars within the M51 system to calculate the distance. The corresponding spatial scale is 47 parsecs per arcsecond.

\section{OBSERVATIONS AND ANALYSIS}

\subsection{Ancillary Data}

The 3.6-4.5-8.0 $\mu \mathrm{m}$ Spitzer (Werner et al. 2004) IRAC (Fazio et al. 2004) data that are shown in Figure 1 were obtained by downloading supermosaics from the Spitzer Heritage Archive. ${ }^{11}$ These data were originally part of the Spitzer Infrared Nearby Galaxy Survey (Kennicutt et al. 2003). The observations were taken in 2004 May, with exposure times of $26.8 \mathrm{~s}$ and were processed through the Spitzer Science Center reduction pipeline, version S18.25.

The Herschel Photo-detecting Array Camera and Spectrometer (PACS; Poglitsch et al. 2010) data were observed in 2009 December in the "blue channel" (70 and $160 \mu \mathrm{m})$ and were downloaded from the Herschel Science Archive (PI: C. Wilson, KPGT_cwilso01; see: Parkin et al. 2013 for the $70 \mu \mathrm{m}$ image). The Level-1 data were reduced using the SCANAMORPHOS (Roussel 2013) package that accurately fits the lowfrequency $(1 / f)$ noise. Typical sky background measurements in the final maps were 0.13 and $0.25 \mathrm{mJy}$ for the 70 and $160 \mu \mathrm{m}$ bands, respectively.

\footnotetext{
${ }^{11}$ http://sha.ipac.caltech.edu/applications/Spitzer/SHA/
} 


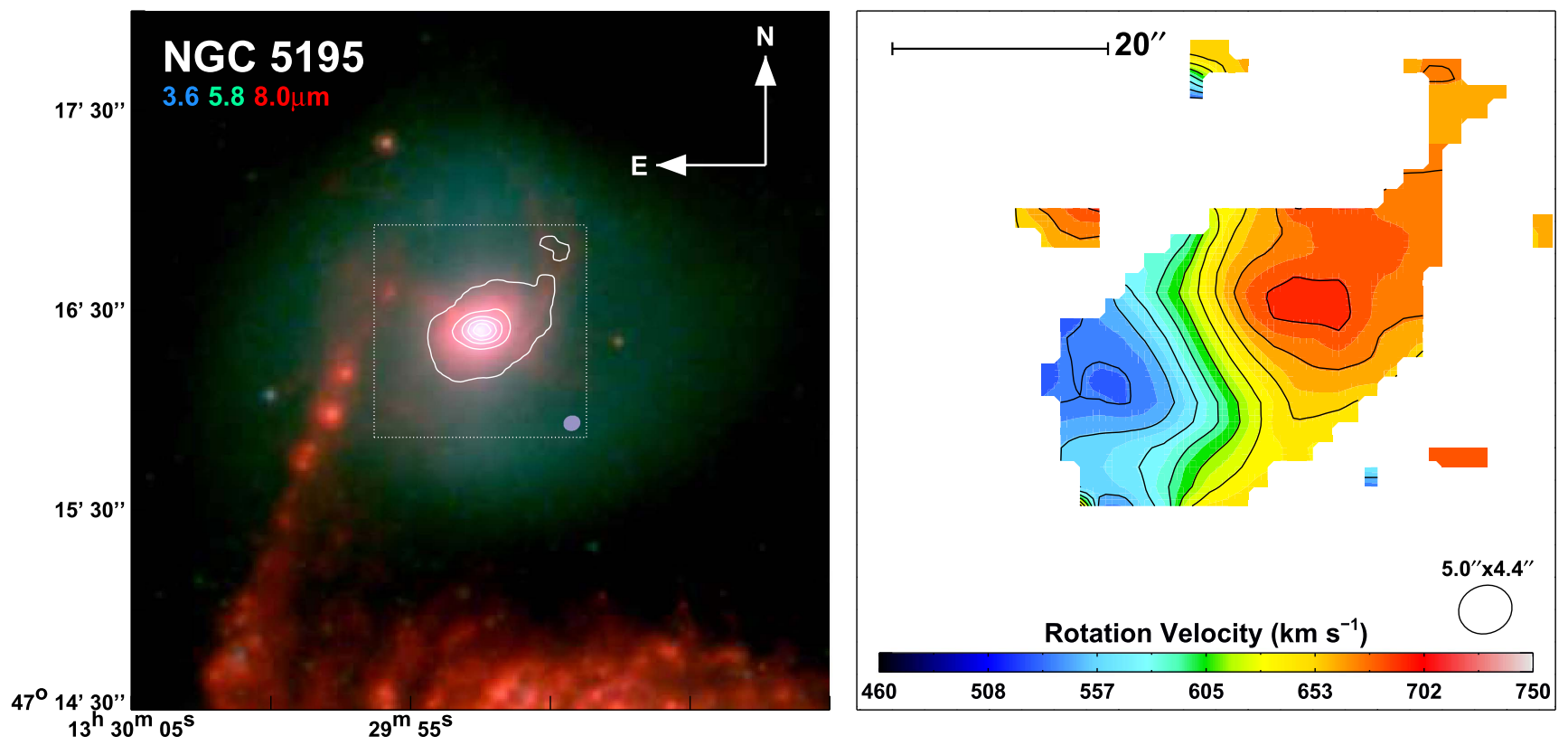

Figure 1. (Left) three-color 3.6, 5.8, 8.0 $\mu \mathrm{m}$ Spitzer IRAC image of NGC 5195 overlaid with the moment0 map for ${ }^{12} \mathrm{CO}(1-0)$ (white contours). The CARMA beam (light purple) is shown at the bottom right of the enclosed box. The red emission represents nonstellar $8.0 \mu \mathrm{m}$ emission, from the $7.7 \mu \mathrm{m}$ PAH feature, with a spiral arm of M51a shown in the foreground. The most prominent PAH emission associated with NGC 5195 is traced by the ${ }^{12} \mathrm{CO}$, including the filament extending to the upper right. (Right) A zoomed-in box of NGC 5195 shows the ${ }^{12} \mathrm{CO}$ moment1 map. All moment1 and channel maps follow the same color scale. The extended filament is also visible in the moment1 map, and on average NGC 5195 appears to follow regular rotation.

The optical spectrum used below was obtained through the Sloan Digital Sky Survey (SDSS), which surveyed galaxies using a dedicated $2.5 \mathrm{~m}$ telescope at Apache Point Observatory. The reduced and calibrated spectral data were obtained from SDSS Data Release 12 (Alam et al. 2015), without any additional modifications applied.

The $1.4 \mathrm{GHz}$ data for NGC 5195, taken as part of the Westerbork SINGS survey (Braun et al. 2007) were downloaded for analysis. Observations of the M51 complex were taken on 2003 June 25 and Nov 23, with a beamsize of $17^{\prime \prime} \times 12$ !" 5 . Because the $1.4 \mathrm{GHz}$ emission associated with NGC 5195 has a complex distribution, we measured the $1.4 \mathrm{GHz}$ flux density from the SINGs image by summing the flux within an aperture matching the extent of the $\mathrm{CO}$ emission using the Common Astronomy Software Application ${ }^{12}$ viewer (McMullin et al. 2007).

\subsection{OSO $20 \mathrm{~m}$ Observations}

$\mathrm{HCN}(1-0)$ and $\mathrm{HCO}^{+}(1-0)$ were observed at the Onsala Space Observatory, Sweden, on 2016 March 3-4, April 15 and 26. The SIS $3 \mathrm{~mm}$ receiver was connected to the Omnisys A spectrometer, which gives a bandwidth $4 \mathrm{GHz}$ wide with dual polarization. The observations were performed with a dualbeam switch mode with a throw of $11^{\prime}$ in azimuth. The half power-beam width of the telescope at the rest frequency $(88.9 \mathrm{GHz})$ was $43^{\prime \prime}$ and the pointing accuracy was better than $3^{\prime \prime}$. The focus was checked each day on bright quasars and the corrections were $\sim 1 \mathrm{~mm}$. The average system temperature was around $150 \mathrm{~K}$, and the opacity 0.2 .

We converted the data from antenna temperature to mainbeam temperature correcting by a beam efficiency of 0.53 . The spectrum was averaged to a final velocity resolution of

\footnotetext{
12 https://casa.nrao.edu/
}

$40 \mathrm{~km} \mathrm{~s}^{-1}(12 \mathrm{MHz})$, for which the achieved rms is $0.145 \mathrm{mK}$. We fitted a baseline of order two to subtract the continuum.

The spectra of the $\mathrm{HCN}$ and $\mathrm{HCO}^{+}$lines are shown in Figure 2. Using the K per Jy factor of 0.07999 , we measure a total $\mathrm{HCN}(1-0)$ line flux of $3.25 \pm 0.27 \mathrm{Jy} \mathrm{km} \mathrm{s}^{-2}$, with velocity width of $219 \mathrm{~km} \mathrm{~s}^{-1}$. We also measure a $\mathrm{HCO}^{+}(1-0)$ total line flux of $2.09 \pm 0.24 \mathrm{Jy} \mathrm{km} \mathrm{s}^{-2}$ with a velocity width of $193 \mathrm{~km} \mathrm{~s}^{-1}$.

We measure an $\mathrm{HCN} / \mathrm{HCO}^{+} J=1-0$ line ratio of 2 . Reproducing $\mathrm{HCN}(1-0) / \mathrm{HCO}^{+}(1-0)$ of 2 with $n=10^{4} \mathrm{~cm}^{-3}$ and $T_{K}=65 \mathrm{~K}$ requires a higher $\mathrm{HCN}$ than $\mathrm{HCO}^{+}$abundance. As long as the emission is not very optically thick the the lower critical density of $\mathrm{HCO}^{+}$would render its $J=1-0$ transition more luminous than that of $\mathrm{HCN}$ (for the same abundance). For the given physical conditions above the $\mathrm{HCN}(1-0)$ / $\mathrm{HCO}^{+}(1-0)$ ratio should be about 0.5 for the same abundance (and for moderate optical depths). To obtain an $\mathrm{HCN}(1-0) /$ $\mathrm{HCO}^{+}(1-0)$ of 2 for $n=10^{4} \mathrm{~cm}^{-3}$ and $T_{K}=65 \mathrm{~K}$, requires the $\mathrm{HCN} / \mathrm{HCO}^{+}$abundance ratio to be about 10 .

\subsection{CARMA Observations}

We present observations of NGC 5195 that were pulled from the CARMA archive, as well as original observations. The archival portion of our NGC 5195 data was observed in ${ }^{12} \mathrm{CO}(1-0)$ with CARMA between 2012 May and December in two different configurations: C-array (1", 30-350 m baselines), D array ( $3^{\prime \prime}, 11-150 \mathrm{~m}$ baselines) by the CARMA \& Nobeyama Nearby Galaxies (Donovan Meyer et al. 2013) survey. We followed up these archival observations in 2014 June with observations of NGC 5195 in E-array (8", 8-66 m baselines), to better detect some of the more diffuse gas that might be present. ${ }^{12} \mathrm{CO}(1-0),{ }^{13} \mathrm{CO}(1-0), \mathrm{CN}\left(1_{0,2}-0_{0,1}\right)$ and $\mathrm{CS}(2-1)$ were observed simultaneously in this set of E-array 


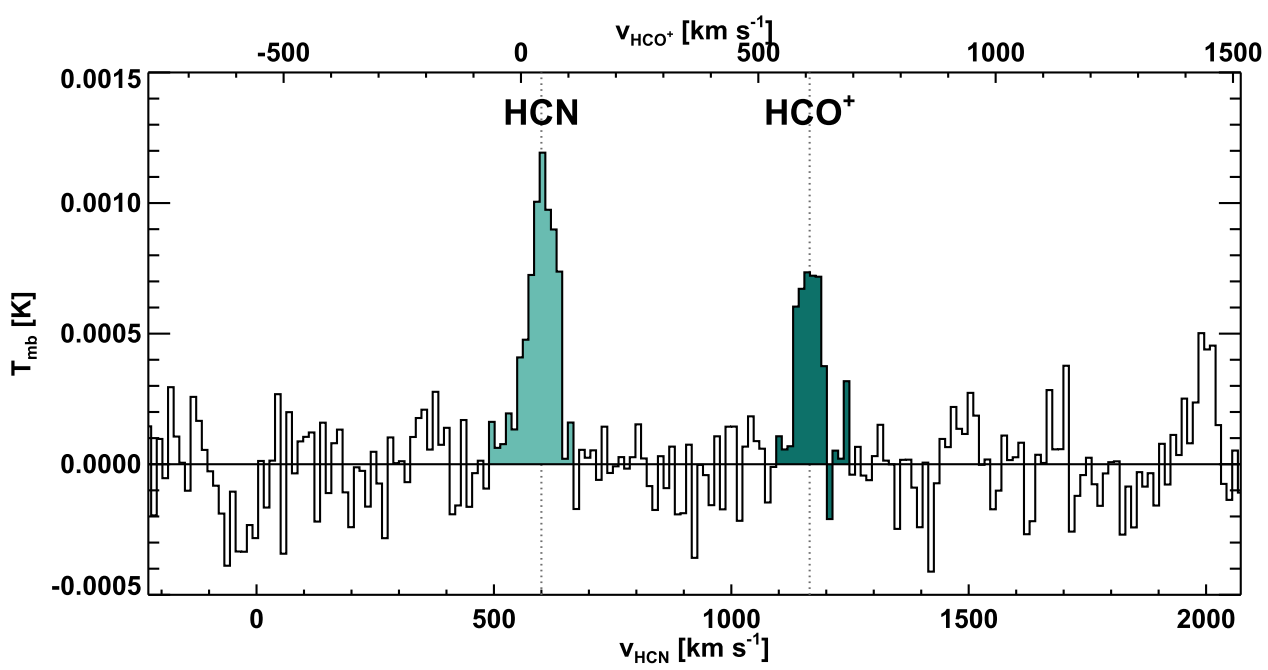

Figure 2. Spectrum of $\mathrm{HCN}(1-0)$ and $\mathrm{HCO}^{+}$from OSO. The lines are robustly detected in the $43^{\prime \prime}$ beam. These confirm the results of Kohno et al. (2002) that the $\mathrm{HCN}$ is weak.

observations, utilizing the upgraded correlator. The observation properties for each molecular line detected by CARMA in NGC 5195 are listed in Table 2. All data were reduced and imaged using the Multichannel Image Reconstruction, Interactive Analysis and Display software (MIRIAD; Sault et al. 1995).

The primary beam has a diameter of $2^{\prime}$ at $\mathrm{CO}(1-0)$, which covers all emission from NGC 5195. M51a itself does appear within the primary beam, but is easily separable from NGC 5195 (see Figure 1(a)). All observations used a long integration on a bright quasar to calibrate the passband, and alternated integrations between a gain calibrator (J1419543) and NGC 5195. We used the MIRIAD task uvlin to separate out continuum emission from the line emission, and we estimate the continuum flux of NGC 5195 to be $1.21 \pm 0.27 \mathrm{mJy}$, centered on $106 \mathrm{GHz}$, from a CARMA image with a 7 "! $6 \times 6$ !" 1 beam. ${ }^{13}$ The calibration and data reduction steps were followed identically to those in Alatalo et al. (2013) to construct channel maps, and moment maps.

Channel maps are shown in Figures 3-6, with the individual gas channels overlaid on a $8 \mu \mathrm{m}$ nonstellar Spitzer IRAC image. To create the underlying Spitzer image, the $8.0 \mu \mathrm{m}$ IRAC4 data was corrected for the stellar contribution by subtracting the $3.6 \mu \mathrm{m}$ IRAC1 image, normalized to the expected stellar continuum at $8 \mu \mathrm{m} \quad(0.232$; Helou et al. 2004). ${ }^{12} \mathrm{CO},{ }^{13} \mathrm{CO}$, and $\mathrm{CN}(1-0)$ are detected. The $\mathrm{CN}(1-0)$ channel map includes blueshifted emission compared to the line peak $\left(\approx 250 \mathrm{~km} \mathrm{~s}^{-1}\right.$ away, at $\left.340 \mathrm{~km} \mathrm{~s}^{-1}\right)$, which we have attributed to M51a and do not consider in subsequent analysis. $\mathrm{CS}(2-1)$ is weak, but is still detected with a signal-tonoise ratio $(\mathrm{S} / \mathrm{N})$ of 3.9. A tentative line, identified as $\mathrm{CH}_{3} \mathrm{CN}$ $\left(6_{k}-5_{k}\right)$, seems to be present in the ${ }^{13} \mathrm{CO}$ spectrum outside of the velocity range that would be inhabited by ${ }^{13} \mathrm{CO}$ in M51a, and has a line flux of $1.27 \pm 0.52$ (only detected with $2.4 \sigma$ significance), but requires deeper observations to confirm whether it is real.

Figures 1 and 7 display the integrated intensity (moment0), and mean velocity (moment1) maps, and Figure 8 shows the integrated spectra of the ${ }^{12} \mathrm{CO},{ }^{13} \mathrm{CO}, \mathrm{CN}(1-0)$, and $\mathrm{CS}(2-1)$

\footnotetext{
${ }^{13}$ This does not include the absolute flux calibration uncertainty of $20 \%$.
}

within NGC 5195, Figure 9 shows the position-velocity (PV) diagrams across the specified slices of the ${ }^{12} \mathrm{CO},{ }^{13} \mathrm{CO}$, and $\mathrm{CN}$ detections (the $\mathrm{CS}(2-1)$ being too low in $\mathrm{S} / \mathrm{N}$ and compact to display). Of note, while the ${ }^{12} \mathrm{CO}$ and ${ }^{13} \mathrm{CO}$ share the same kinematic axis (of $30^{\circ}$ ), the $\mathrm{CN}$ (with an angle of $0^{\circ}$ ) has a different kinematic major axis from the other gas tracers and is thus considered kinematically misaligned according to Davis et al. (2011).

The integrated spectrum for each line was constructed using the moment 0 map to create a clip-mask and integrating the flux within the moment0-defined (unmasked) aperture in each channel of the data cube. This was done separately for each tracer. The rms noise was then calculated as the standard deviation of all pixels in the cube outside of the moment0aperture per channel and is listed in Table 2. An additional $30 \%$ correction was also added in quadrature to the rms noise to account for the oversampling of the maps (see: Alatalo et al. 2015b for details). The rms noise per channel for the spectrum was then calculated by multiplying the rms of the entire data cube by the square root of the total number of beams in the moment 0 -aperture.

The line luminosity for each molecule was calculated using Solomon \& Vanden Bout (2005):

$$
L_{\text {line }}=1.04 \times 10^{-3} S_{\text {line }} \Delta v \nu_{\text {rest }}(1+z)^{-1} D_{L}^{2},
$$

where $S_{\text {line }} \Delta v$ is the line flux in $\left[\mathrm{Jy} \mathrm{km} \mathrm{s}^{-1}\right], \nu_{\text {rest }}$ is the rest frequency of the line in $[\mathrm{GHz}], z$ is the redshift of NGC 5195 (calculated using a systemic velocity of $634 \mathrm{~km} \mathrm{~s}^{-1}$, the $\mathrm{CO}(1-0)$ spectrum), and $D_{L}$ is the luminosity distance, defined as $9.9 \mathrm{Mpc}$ in Section 1. The resultant line fluxes are listed in solar luminosities in Table 2.

\subsection{Comparison to Previous Detections}

Kohno et al. (2002) observed ${ }^{12} \mathrm{CO}(1-0)$ and $\mathrm{HCN}(1-0)$ in NGC 5195 with the NRO $45 \mathrm{~m}$ single dish, and imaged the ${ }^{12} \mathrm{CO}(1-0)$ with the NMA Interferometer. The new OSO HCN (1-0) observations are consistent with the relatively faint $\mathrm{HCN}$ emission found by Kohno et al. (2002). Using the $45 \mathrm{~m}$, Kohno et al. (2002) find a total line flux of $199 \mathrm{Jy} \mathrm{km} \mathrm{s}^{-1}$ (using a 2.4 Jy K ${ }^{-1}$ conversion; Ueda et al. 2014), in conflict with their 
Table 2

Derived Molecular Properties

\begin{tabular}{|c|c|c|c|c|c|}
\hline & & ${ }^{12} \mathrm{CO}(1-0)$ & ${ }^{13} \mathrm{CO}(1-0)$ & $\overline{\mathrm{CN}}(1-0)$ & $\overline{\mathrm{CS}(2-1)}$ \\
\hline$\nu_{\text {rest }}$ & $(\mathrm{GHz})$ & 115.2712 & 110.2014 & 113.4910 & 97.9810 \\
\hline$\theta_{\text {beam }}$ & $\left({ }^{\prime \prime}\right)$ & $5.0 \times 4.4$ & $7.4 \times 5.9$ & $7.2 \times 5.8$ & $8.3 \times 6.7$ \\
\hline $\mathrm{K} \mathrm{Jy}^{-1}$ & $\ldots$ & 4.126 & 2.290 & 2.291 & 2.290 \\
\hline$v_{\text {range }}$ & $\left(\mathrm{km} \mathrm{s}^{-1}\right)$ & $463-799$ & $448-729$ & $298-740$ & $473-674$ \\
\hline$\Delta v$ & $\left(\mathrm{~km} \mathrm{~s}^{-1}\right)$ & 10 & 20 & 40 & 50 \\
\hline $\mathrm{rms}$ & $\left(\mathrm{mJy} \mathrm{bm}^{-1}\right)$ & 12.0 & 3.3 & 4.7 & 4.0 \\
\hline$I_{\text {peak }}$ & $\left(\mathrm{K} \mathrm{km} \mathrm{s}^{-1}\right)$ & $243.8 \pm 15.1$ & $23.9 \pm 2.3$ & $19.6 \pm 2.3$ & $5.7 \pm 1.1$ \\
\hline \multirow[t]{2}{*}{ Mom0 area } & $(\operatorname{arcseconds})^{\mathrm{a}}$ & 1489 & 763 & 365 & 360 \\
\hline & $\left(\mathrm{kpc}^{2}\right)$ & 3.29 & 1.69 & 0.81 & 0.80 \\
\hline rms/channel & (mJy) & 97.3 & 13.0 & 13.2 & 9.6 \\
\hline Line flux & $\left(\mathrm{Jy} \mathrm{km} \mathrm{s}^{-1}\right)$ & $243.8 \pm 5.2$ & $22.7 \pm 1.0$ & $12.4 \pm 1.4$ & $3.9 \pm 1.0$ \\
\hline $\mathrm{S} / \mathrm{N}$ (Line) & $\cdots$ & 46.9 & 22.7 & 8.9 & 3.9 \\
\hline$L_{\text {line }}$ & $\left(L_{\odot}\right)$ & $2859 \pm 61$ & $255 \pm 11$ & $143 \pm 16$ & $39 \pm 10$ \\
\hline
\end{tabular}

Note.

${ }^{a} \operatorname{arcsec}^{2}$.

resultant interferometric flux of $340 \mathrm{Jy} \mathrm{km} \mathrm{s}^{-1}$. We have measured a ${ }^{12} \mathrm{CO}(1-0)$ line flux of $243.8 \pm 5.2 \mathrm{Jy} \mathrm{km} \mathrm{s}^{-1}$, falling right between the two estimates of those authors (34\% larger than the $45 \mathrm{~m}$, and $27 \%$ lower than the NMA line fluxes). Were some of the M51a emission to be included in the map, it would result in an overestimation of the $\mathrm{CO}(1-0)$ line flux for NGC 5195, although Kohno et al. (2002) make note of the lowvelocity component likely being from the spiral arm of NGC 5194 (displayed as gray contours in the first nine channels in Figure 3). If we also account for the $20 \%$ absolute flux uncertainties associated with the $3 \mathrm{~mm}$ flux calibration (for both the NMA and the CARMA observations), we consider ourselves to be in reasonable agreement with Kohno et al. (2002).

Sage (1990) presented ${ }^{12} \mathrm{CO}(2-1)$ and ${ }^{13} \mathrm{CO}(1-0)$ observations of NGC 5195 from the NRAO $12 \mathrm{~m}$, measuring a ${ }^{13} \mathrm{CO}$ line flux of $12.5 \pm 3.3 \mathrm{Jy} \mathrm{km} \mathrm{s}^{-1}$ (using the $\mathrm{Jy} / \mathrm{K}=30.4$ of the NRAO 12m; Ueda et al. 2014). We have detected nearly twice the total ${ }^{13}$ CO flux of Sage (1990), although that is likely due to $\mathrm{S} / \mathrm{N}$ (our detection has six times higher $\mathrm{S} / \mathrm{N}$ than Sage 1990). It is also possible that pointing errors and calibration uncertainties might also play a part. Matsushita et al. (2010) used the NRO $45 \mathrm{~m}$ to measure the ${ }^{13} \mathrm{CO}(1-0)$ of NGC 5195 to be $28.8 \mathrm{Jy} \mathrm{km} \mathrm{s}^{-1}$, in much better agreement (within 22\%) with our ${ }^{13} \mathrm{CO}$ measurement. Overall, our observations are within reasonable agreement with those done previously.

\subsection{Molecular Mass and $\mathrm{H}_{2}$ Surface Density in NGC 5195}

We calculate the molecular mass expected in NGC 5195 using the ${ }^{12} \mathrm{CO}(1-0)$ luminosity. Assuming that NGC 5195 is similar to the Milky Way, where we assume that molecular gas is distributed within giant molecular clouds, the conversion between the $\mathrm{CO}$ luminosity and the $\mathrm{H}_{2}$ mass (from Bolatto et al. 2013) is:

$$
\frac{M_{\mathrm{H}_{2}}}{L_{\mathrm{CO}}}=8.75 \times 10^{4} \frac{M_{\odot}}{L_{\odot}},
$$

which corresponds to a $X_{\mathrm{CO}}$ conversion factor of $2 \times 10^{20} \mathrm{~cm}^{-2}\left(\mathrm{~K} \mathrm{~km} \mathrm{~s}^{-1}\right)^{-1}$, considered the standard, holding both for the Milky Way and most normal nearby galaxies.
Using this conversion, and $L_{\mathrm{CO}}{ }^{14}$ from Table 2, we find a total $\mathrm{H}_{2}$ mass of $(2.74 \pm 0.06) \times 10^{8} M_{\odot} \cdot{ }^{15}$

To calculate the $\mathrm{H}_{2}$ surface density, $\Sigma_{\mathrm{H}_{2}}$, we use the area subtended by the ${ }^{12} \mathrm{CO}(1-0)$ clipped moment map (listed in Table 2), converted from arcseconds to the physical scale of the galaxy to be $3.15 \mathrm{kpc}^{2}$, which corresponds to a mean $\mathrm{H}_{2}$ surface density, $\left\langle\Sigma_{\mathrm{H}_{2}}\right\rangle=87 \pm 2 M_{\odot} \mathrm{pc}^{-2}$. To calculate the peak $\Sigma_{\mathrm{H}_{2}}$, we convert the peak line intensity to the $\mathrm{H}_{2}$ surface density, assuming the conversion from Bolatto et al. (2013):

$$
\frac{\Sigma_{\mathrm{H}_{2}}}{M_{\odot} \mathrm{pc}^{-2}}=\frac{3.16 I_{\text {peak }}}{\mathrm{K} \mathrm{km} \mathrm{s}^{-1}}
$$

where $I_{\text {peak }}$ represents the peak intensity of the ${ }^{12} \mathrm{CO}$ map, resulting in $\Sigma_{\mathrm{H}_{2} \text {,peak }}=810 \pm 50 M_{\odot} \mathrm{pc}^{-2}$, made over an area of $0.05 \mathrm{kpc}^{2}$ (one beam), an order of magnitude larger than $\left\langle\Sigma_{\mathrm{H}_{2}}\right\rangle$, and consistent with the range seen in nearby disk galaxies (Bigiel et al. 2008; Leroy et al. 2008).

\section{THE STATE OF THE MOLECULAR GAS IN NGC 5195}

\subsection{The Revised ${ }^{13} \mathrm{CO} /{ }^{12} \mathrm{CO}$ Ratio}

Sage (1990) measured the ${ }^{12} \mathrm{CO}$ and ${ }^{13} \mathrm{CO}$ in NGC 5195 (discussed in Section 2.4), finding a ${ }^{12} \mathrm{CO} /{ }^{13} \mathrm{CO}$ flux ratio (hereafter, $\mathcal{R}_{12 / 13}$ ) to be $\approx 26$. This high ratio is often seen in hot dust hosts and ULIRGs (Aalto et al. 1995), suggestive of a hot, disrupted molecular disk in which the ${ }^{12} \mathrm{CO}$ emission includes a large optically thin contribution. The CARMAderived ${ }^{12} \mathrm{CO}$ and ${ }^{13} \mathrm{CO}$ fluxes shown in Table 2 update $\mathcal{R}_{12 / 13}$ in NGC 5195 to be $11.4 \pm 0.5$, which is more consistent with normal star-forming galaxies as well as field early-type galaxies (Alatalo et al. 2015b) than interacting galaxies. The revised $\mathcal{R}_{12 / 13}$ in NGC 5195 seems to suggest that the dynamical state of the gas is not as disrupted as originally thought, having had time to settle since the last interaction.

\footnotetext{
${ }^{14}$ The CO line luminosity.

15 Does not include the $30 \%$ mass conversion uncertainty (Bolatto et al. 2013) or $20 \%$ absolute flux uncertainty.
} 


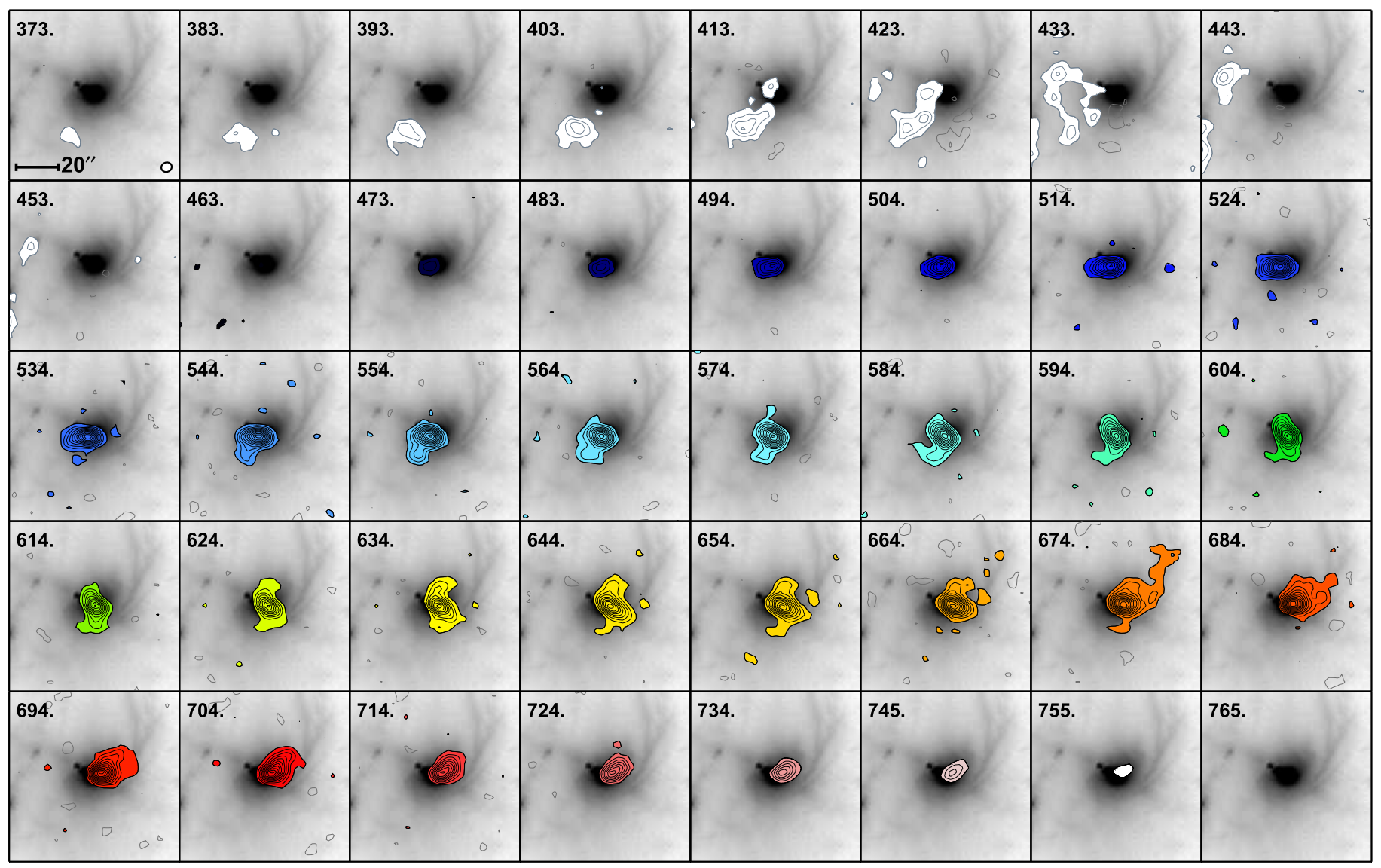

Figure 3. ${ }^{12} \mathrm{CO}(1-0)$ channel maps of NGC 5195 from CARMA (filled contours) overlaid on the nonstellar $8.0 \mu \mathrm{m}$ maps from Spitzer (grayscale), tracing the PAH emission. The contours are color coded based on the relative red- or blueshift of the channel. The contours start at $3 \sigma$ (with $\sigma$ representing the rms noise in Table 2), placed at $3 \sigma$ intervals. Negative contours (dark gray) are $[-3,-6,-9] \sigma$. Channels with ${ }^{12} \mathrm{CO}$ emission assumed to be associated with the M51a spiral arm contain a white fill and gray contours. The velocity color scale is identical to Figure 1. The velocities listed in the top left are optical recession velocities. The extension into the PAH filament can be seen between 634 and $694 \mathrm{~km} \mathrm{~s}^{-1}$.

\subsection{What is CN Tracing?}

The $\mathrm{CN}$ abundance can be enhanced either by UV fields (for example as a photo-dissociation product of $\mathrm{HCN}$ in the envelopes of molecular clouds) or by X-rays that can penetrate further into the inner, denser regions. Hence, $\mathrm{CN}$ is a molecular tracer of photo-dissociated regions (PDRs) and X-ray dissociated regions (XDRs), depending on the environment (e.g., Lepp \& Dalgarno 1996; Rodriguez-Franco et al. 1998). The strong PAH, IR, and $\mathrm{H} \alpha$ emission in NGC 5195 (Boulade et al. 1996; Greenawalt et al. 1998 and Figure 1) suggest that the $\mathrm{CN}$ is associated with the old starburst caused by the encounter with M51a and may be associated with PDRs.

To explore the possibility that $\mathrm{CN}$ could be tracing XDRs, we compared our $\mathrm{CN}$ intensity maps with the X-ray emission from Schlegel et al. (2016), where Chandra images show two recent outbursts coming out from the supermassive black hole in NGC 5195. A comparison of the CN and X-ray images shows no obvious spatial correlation between them.

In a barred galaxy potential, dynamical models predict two kinds of orbits: (1) $x 1$ orbits oriented parallel to the major axis of the bar that support the bar and (2) $x 2$ orbits aligned perpendicular to the bar and located closer to a galaxy's nucleus. Gas in self-intersecting $x 1$ orbits tends to shock and lose energy, eventually changing orientation and moving inward to the lower-energy $x 2$ orbits. For a review, we refer readers to Alloin (2006). The moment maps in Figure 7 show that $\mathrm{CN}$ arises from the inner regions of the galaxy with isovelocity contours at a different angle than those of ${ }^{12} \mathrm{CO}$ and ${ }^{13} \mathrm{CO}$. The latter seem to trace a more extended gas distribution, possibly associated with the $x 1$ orbits of the large-scale bar (Kohno et al. 2002), while $\mathrm{CN}$ could be tracing the perpendicular $x 2$ orbits of the inner region. It is possible that this difference in alignment is due to resolution effects, but given the high S/N CN channels seen in Figure 5, we believe the detection of differing alignments is robust.

This would be a similar scenario to that observed in the central $100 \mathrm{pc}$ of $\mathrm{M} 82$, where the $\mathrm{CN}$ abundance (among other PDR tracers) is enhanced by a factor of 3 in the $x 2$ orbits (Ginard et al. 2015). This result is consistent with NGC 5195 being a postburst system, with forbidden neon lines present in the IR spectrum (Boulade et al. 1996) and the optical lines ( $\mathrm{H} \alpha$ absorption and strong [N II] emission; Greenawalt et al. 1998) in the nucleus, but with little ionized gas in the outskirts. Deep IFU imaging of the optical lines ( $\mathrm{H} \alpha$ and [N II]) will be able to confirm this scenario.

\subsection{Molecular Line Ratios}

We assume that the HCN emission is emerging from the same region as the $\mathrm{CN}$ emission and then estimated the line ratio by converting the CARMA flux to the appropriate 


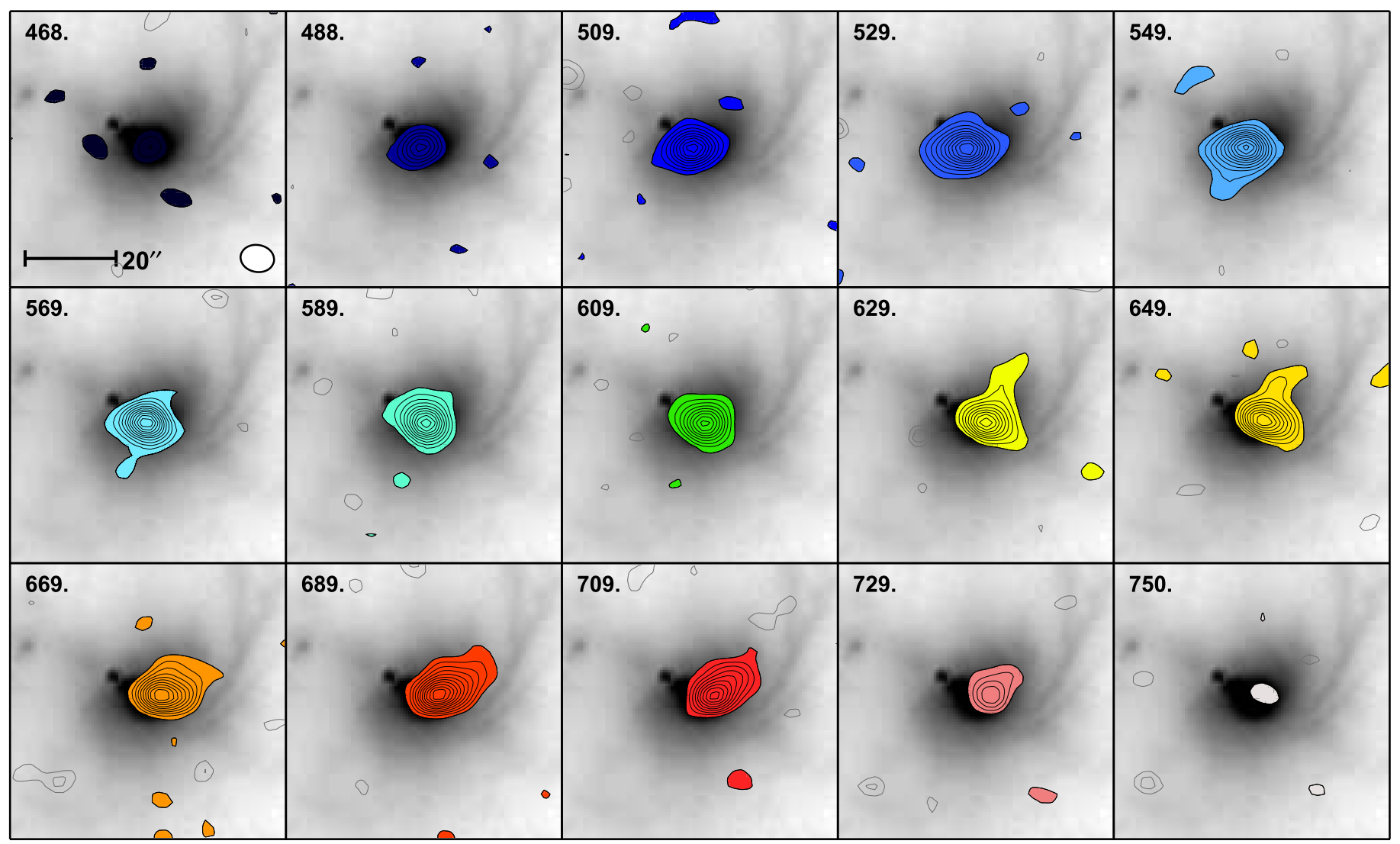

Figure 4. ${ }^{13} \mathrm{CO}(1-0)$ channel maps of NGC 5195 from CARMA (filled contours) overlaid on the nonstellar $8.0 \mu \mathrm{m}$ maps from Spitzer (grayscale), tracing the PAH emission. These contours are color coded based on the relative red- or blueshift of the channel, placed at $2 \sigma$ intervals starting at $3 \sigma$. The velocities listed in the top left are optical recession velocities. The velocity color scale is identical to Figure 1. A slight extension is seen along the PAH filament in ${ }^{13} \mathrm{CO}(1-0)$, but given the relative faintness of ${ }^{13} \mathrm{CO}$ compared to ${ }^{12} \mathrm{CO}$, it is likely that the more extended structure is below our detection threshold.

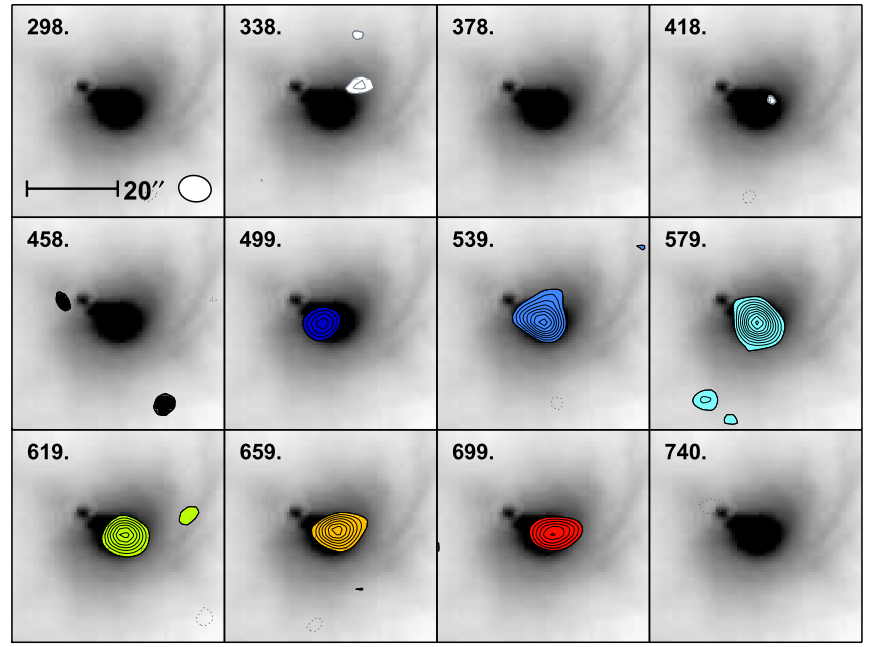

Figure 5. CN(1-0) channel maps of NGC 5195 from CARMA (filled contours) overlaid on the nonstellar $8.0 \mu \mathrm{m}$ maps from Spitzer (grayscale), tracing the PAH emission. The contours are color coded based on the relative red- or blueshift of the channel, placed at $1 \sigma$ intervals starting at $3 \sigma$. The velocities listed in the top left are optical recession velocities. The velocity color scale is identical to Figure 1. $\mathrm{CN}$ is well detected in individual channels.

brightness temperature for the NRO $45 \mathrm{~m}$. We followed the same procedure for $\mathrm{CS}(2-1)$. We find line ratios of $\mathrm{HCN}(1-0) /$ $\mathrm{CS}(2-1)=1.2, \mathrm{CN}(1-0) / \mathrm{HCN}(1-0)=2.1$ and $\mathrm{CN}(1-0) / \mathrm{CS}$ $(2-1)=3.3$. Comparing different transitions of different

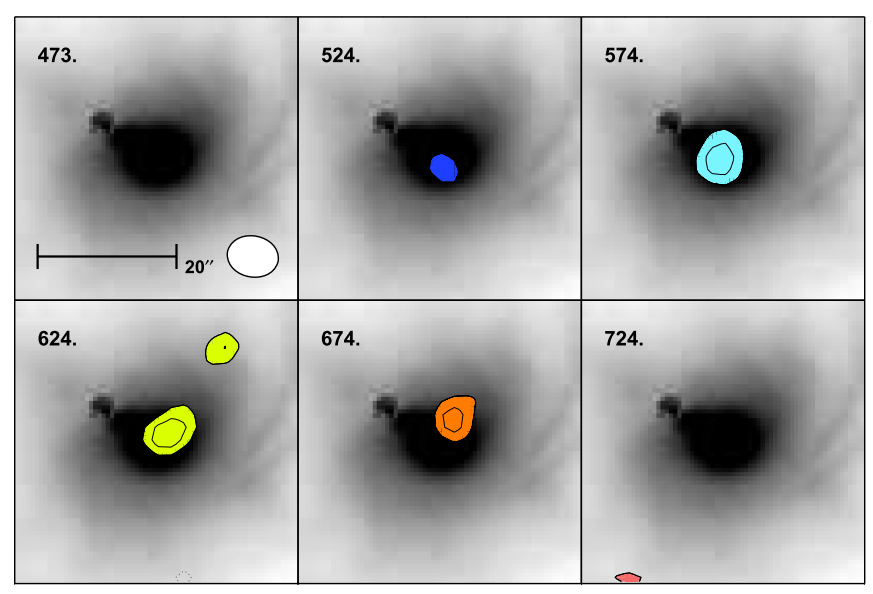

Figure 6. CS(2-1) channel maps of NGC 5195 from CARMA (filled contours) overlaid on the nonstellar $8.0 \mu \mathrm{m}$ maps from Spitzer (grayscale), tracing the $\mathrm{PAH}$ emission. The contours are color coded based on the relative red- or blueshift of the channel, placed at $1 \sigma$ intervals starting at $3 \sigma$. The velocities listed in the top left are optical recession velocities. The velocity color scale is identical to Figure 1 . $\mathrm{CS}(2-1)$ is tentatively $(\mathrm{S} / \mathrm{N} \approx 3-4)$ detected in individual channels, but the total line is robustly detected.

species is not ideal, because one is tracing a mixture of excitation and chemistry. In spite of this, it seems safe to say that $\mathrm{CN}$ is more abundant than $\mathrm{HCN}$ and $\mathrm{CS}$ in the central $\mathrm{kpc}$ of NGC 5195. 


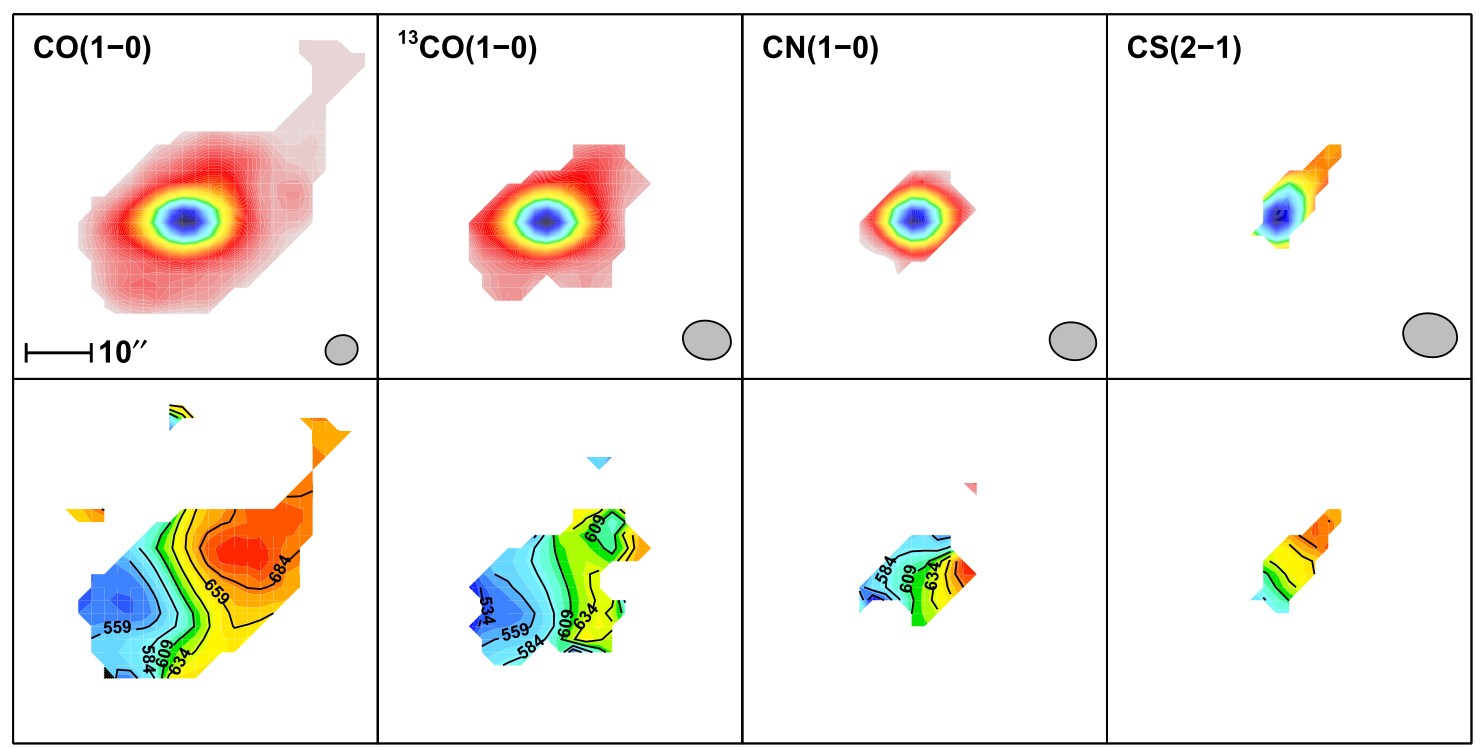

Figure 7. Moment maps of all detected molecules, including ${ }^{12} \mathrm{CO}(1-0),{ }^{13} \mathrm{CO}(1-0), \mathrm{CN}(1-0)$, and $\mathrm{CS}(2-1)$. The top row displays the clipped integrated intensity maps of each molecular species detected, and the bottom row displays the mean velocity maps of each species. The velocity color scale is identical to Figure 1 . The CS(2-1), the faintest line, traces only the core of the molecular gas in NGC 5195, which supports the idea that the central region contains the densest gas. The ${ }^{12} \mathrm{CO}$, ${ }^{13} \mathrm{CO}$, and CS trace similar velocity structures, whereas the velocity map of the $\mathrm{CN}$ seems to trace different kinematics.

Using RADEX (van der Tak et al. 2007), ${ }^{16}$ and assuming a gas kinetic temperature of $65 \mathrm{~K}$ (from the dust temperature derived by Smith 1982), an HCN/CS ratio of about unity can be obtained with volume densities of about $10^{4} \mathrm{~cm}^{-3}$ and assuming similar $\mathrm{HCN}$ and $\mathrm{CS}$ abundances. This may point to a very low fraction of dense gas (i.e., gas with number density $n \gtrsim 10^{5} \mathrm{~cm}^{-3}$ ) that is in agreement with the emission of $\mathrm{CN}$ and CS, as well as with the high ratio $\mathrm{CO}(1-0) / \mathrm{HCN}(1-0)$ of 85.3. ${ }^{17}$

Assuming that $\mathrm{CN}$ is tracing PDRs in NGC 5195, the UV fields created by the low star formation in the galaxy (see Section 4) could dissociate and fragment the molecular clouds, creating larger envelopes in which most of the $\mathrm{CN}$ would form. The $\mathrm{CN}(1-0)$ line is brighter than that of $\mathrm{HCN}$. The radiative transfer of $\mathrm{CN}$ is not straightforward, but if we assume collisional excitation, the line ratio indicates higher $\mathrm{CN}$ abundances that that of HCN. Since CN can be a photodissociation product of $\mathrm{HCN}$, this may be the result of UV irradiation of fragmented dense clumps. However, one would then also expect to see a strong $\mathrm{HCO}^{+}$line. There are some star-forming regions with $\mathrm{X}(\mathrm{CN})>\mathrm{X}(\mathrm{HCN})>\mathrm{X}\left(\mathrm{HCO}^{+}\right)$in (for example) the Orion ridge (Blake et al. 1986), but instead this ratio may emerge from the region around an AGN. Lepp \& Dalgarno (1996) suggested that $\mathrm{X}(\mathrm{CN})>\mathrm{X}(\mathrm{HCN})$ in $\mathrm{X}$-ray irradiated regions. It is possible that (at least a significant fraction of) the high density tracer emission is emerging from the nucleus instead of being scattered in a poststarburst region.

Apart from the species previously mentioned, we marginally detect $\mathrm{CH}_{3} \mathrm{CN}\left(6_{k}-5_{k}\right)$ (located between 200 and $350 \mathrm{~km} \mathrm{~s}^{-1}$ at the red side of ${ }^{13} \mathrm{CO}$ in Figure 8). Its integrated flux, of 1.3 Jy $\mathrm{km} \mathrm{s}^{-1}$ is, however, below a $3 \sigma$ detection. This molecule is

\footnotetext{
16 http://home.strw.leidenuniv.nl/ moldata/radex.html

17 A $\mathrm{HCN}(1-0) / \mathrm{CS}(2-1)$ line ratio close to unity may also emerge from an even denser gas component with $n>10^{4} \mathrm{~cm}^{-3}$, but this would require that the $\mathrm{CS}$ abundances exceed those of HCN. We assume collisional excitation only, no significant optical depth effects, and that the hyperfine structure lines of HCN(1-0) can simply be added (see discussion in Aalto et al. 2015).
}

also a tracer of dense gas $\left(n_{\text {crit }}\left[\mathrm{CH}_{3} \mathrm{CN}\left(6_{k}-5_{k}\right)\right] \sim\right.$ $\left.10^{5} \mathrm{~cm}^{-3}\right)$, similar to those of $\mathrm{CN}(1-0)$ and $\mathrm{CS}(2-1)$. However, $\mathrm{CH}_{3} \mathrm{CN}$ is easily dissociated by UV fields (Aladro et al. 2013), and thus it typically resides in the cores of the molecular clouds. Based on that, the ratio between $\mathrm{CN}$ and $\mathrm{CH}_{3} \mathrm{CN}$ would be a rough indicator of the amount of dense gas residing in the envelopes versus the inner parts. We obtain a tentative ratio of $\mathrm{CN} / \mathrm{CH}_{3} \mathrm{CN}=9.0$. Interestingly, the circumnuclear regions of the Seyfert galaxies NGC 1097 and NGC 1068 (Aladro et al. 2013; Martí et al. 2015) show luminous $\mathrm{CH}_{3} \mathrm{CN}$ emission. High-resolution observations will reveal if NGC 5195 also has a circumnuclear region of dense gas irradiated by an AGN. Such a structure may have a complex chemistry with suppressed $\mathrm{HCO}^{+}$while $\mathrm{CN}$ abundances can be high and also those of $\mathrm{CH}_{3} \mathrm{CN}$ in warm, shielded regions.

\section{PAST AND PRESENT STAR FORMATION IN NGC 5195}

\subsection{The Stellar Populations of NGC 5195}

In order to classify the stellar population of NGC 5195, we used the Penalized Pixel Fitting software (PPXF; Cappellari \& Emsellem 2004) ${ }^{18}$ applied to the nuclear spectrum $^{19}$ of NGC 5195 from the SDSS DR12 (Alam et al. 2015). The 3" spectral fiber is ideal for this study, as spectra of NGC 5195 that are more extended than the nuclear region tend to be contaminated by the spiral arm of M51a (see: Figure 13.64 of Moustakas et al. 2010). Integral field spectroscopy would be able to differentiate between NGC 5195 and M51a, to gain a clearer picture of the integrated stellar population properties of NGC 5195, but is beyond the scope of this paper.

We used the stellar templates from the MILES library (Vazdekis et al. 2010), normalized to a uniform (Salpeter 1955)

\footnotetext{
18 http://www-astro.physics.ox.ac.uk/ mxc/software/\#ppxf

19 http://data.sdss3.org/spectrumDetail?

$\mathrm{mjd}=53063 \&$ fiber $=527 \&$ plateid $=1463$
} 


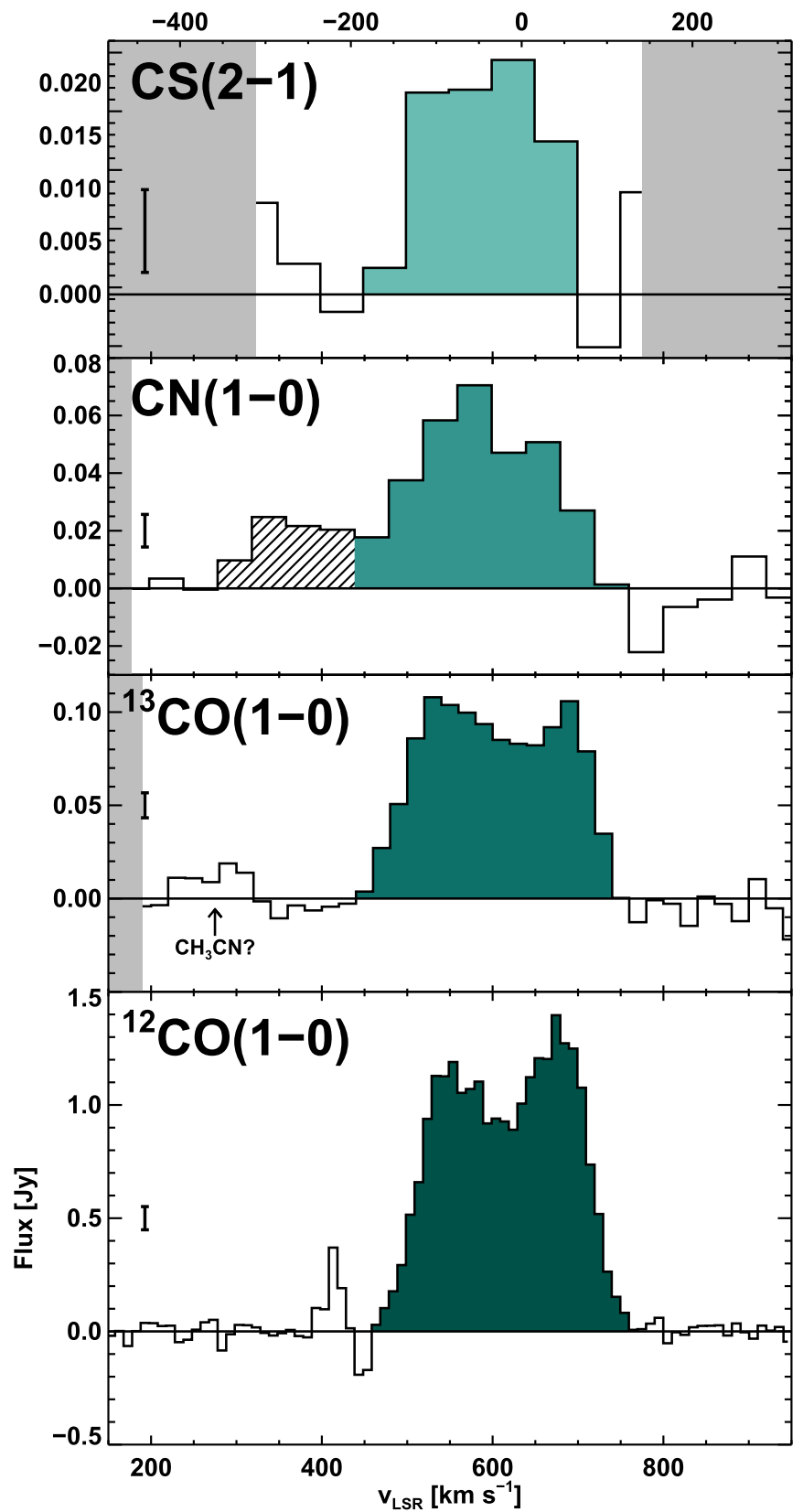

Figure 8. Extracted spectra of the CARMA-detected molecular lines. The moment0 maps were used as masks to integrate the data cubes to create the spectra of $\mathrm{CS}(2-1)$ (top), $\mathrm{CN}(1-0)$ (top middle), ${ }^{13} \mathrm{CO}(1-0)$ (bottom middle) and ${ }^{12} \mathrm{CO}(1-0)$ (bottom). The position of the tentatively identified $\mathrm{CH}_{3} \mathrm{CN}$ is shown in the ${ }^{13} \mathrm{CO}(1-0)$ spectrum. The error bar at the left of each panel marks the $1 \sigma$ noise in the spectrum. The shaded gray regions represent the edges of the correlator bands used for each of the spectra. The $\mathrm{CN}(1-0)$ spectrum shows emission that does not appear to be associated with the $\mathrm{CN}(1-0)$ line in NGC 5195 (see Figure 5), and is shaded with diagonal lines.

initial mass function (IMF), using models representing 50 stellar ages from $0.06-17 \mathrm{Gyr}$, and four metallicities $(-0.71<Z<0.22)$, for a total of 200 models. We included regularization within PPXF (regul $=0.004$ ) to allow us to understand the range of possible stellar populations that would match the nuclear spectrum. Figure 10 shows the model fit to the central spectrum of NGC 5195, and Figure 11 shows the probability distribution function of the PPXF model fitting, summed over the metallicity axis. The nucleus of NGC 5195 clearly shows two stellar populations: $80 \%$ of nuclear stars are old ( $\gtrsim 10 \mathrm{Gyr}$ ) and $20 \%$ are of intermediate age ( $\approx 1 \mathrm{Gyr})$, consistent with A-stars.

As Figure 11 shows, there is some uncertainty to the age of the intermediate-aged stellar population, mostly based on degeneracies of metallicity, but also likely because the SDSS fiber only subtends a small portion (144 pc) of the nucleus. This does put a significant constraint on the interaction that took place between NGC 5195 and M51a, suggesting when enhanced star formation was present in NGC 5195. Given that star formation is known to increase even at large radii during gravitational encounters (Scudder et al. 2012; Moreno et al. 2015), it is likely that this stellar population represents the enhanced star formation that was taking place throughout the encounter (which was suggested to take place $\approx 1 / 2 \mathrm{Gyr}$ ago; Salo \& Laurikainen 2000; Dobbs et al. 2010). The spectral stellar population fit also agrees within errors with the photometrically derived stellar populations (Mentuch Cooper et al. 2012), and might provide another constraint to model the complex history of interaction that has occurred in the M51 system.

\subsection{Integrated Star Formation}

The SFR of NGC 5195 was calculated by Lanz et al. (2013) using MAGPHYS (da Cunha et al. 2008) to model the far-UV to far-IR SED, measuring it to be $0.142 M_{\odot} \mathrm{yr}^{-1}$. To estimate the contribution of the M51a to the Lanz et al. (2013) SFR, we defined a region based on the $8 \mu \mathrm{m}$ PAH emission from the foreground arm and measured the 70 and $8 \mu \mathrm{m}$ photometry excluding the region. We find that $8 \%$ of the PACS $70 \mu \mathrm{m}$ flux and $18 \%$ of the IRAC $8 \mu \mathrm{m}$ flux are contained in the excluded region. While this region may also contain some emission from NGC 5195, the strong correlation of the luminosities in these bands to SFR enable us to estimate that $10 \%-20 \%$ of the Lanz et al. (2013) SFR may be due to M51a, revising the integrated SFR down to $0.116 M_{\odot} \mathrm{yr}^{-1}$.

Using the revised SFR, and dividing by the moment 0 area from Table 2 , we derive an SFR rate surface density $\Sigma_{\mathrm{SFR}} \approx$ $0.105 M_{\odot} \mathrm{yr}^{-1} \mathrm{kpc}^{-2}$ (renormalized to our chosen distance to NGC 5195 and use of a Salpeter initial mass function; Salpeter 1955).

To determine if the molecular gas is inefficient globally, we investigate where NGC 5195 fits on the Schmidt-Kennicutt (S-K) star-formation surface density-gas surface density relation (Schmidt 1959; Kennicutt 1998; Kennicutt \& Evans 2012):

$$
\frac{\Sigma_{\mathrm{SFR}}}{M_{\odot} \mathrm{yr}^{-1} \mathrm{kpc}^{-2}}=2.5 \times 10^{-4}\left(\frac{\Sigma_{\mathrm{H}_{2}}}{M_{\odot} \mathrm{pc}^{-2}}\right)^{1.4} .
$$

If we use the average molecular surface density calculated from our ${ }^{12} \mathrm{CO}$ observations, $\left\langle\Sigma_{\mathrm{H}_{2}}\right\rangle=87 \pm 2 M_{\odot} \mathrm{pc}^{-2}$, we predict an average $\Sigma_{\mathrm{SFR} \text {,predicted }} \approx 0.13 M_{\odot} \mathrm{yr}^{-1} \mathrm{kpc}^{-2}$, in good agreement with the measured $\Sigma_{\mathrm{SFR}}$. We, therefore, conclude that the global molecular gas in NGC 5195 is efficiently forming stars. Two caveats to this analysis are (1) that the Lanz et al. (2013) star formation could have been contaminated by the spiral arm in M51a (seen in PAH emission in Figure 1) and (2) that the intermediate-aged stellar population could be 

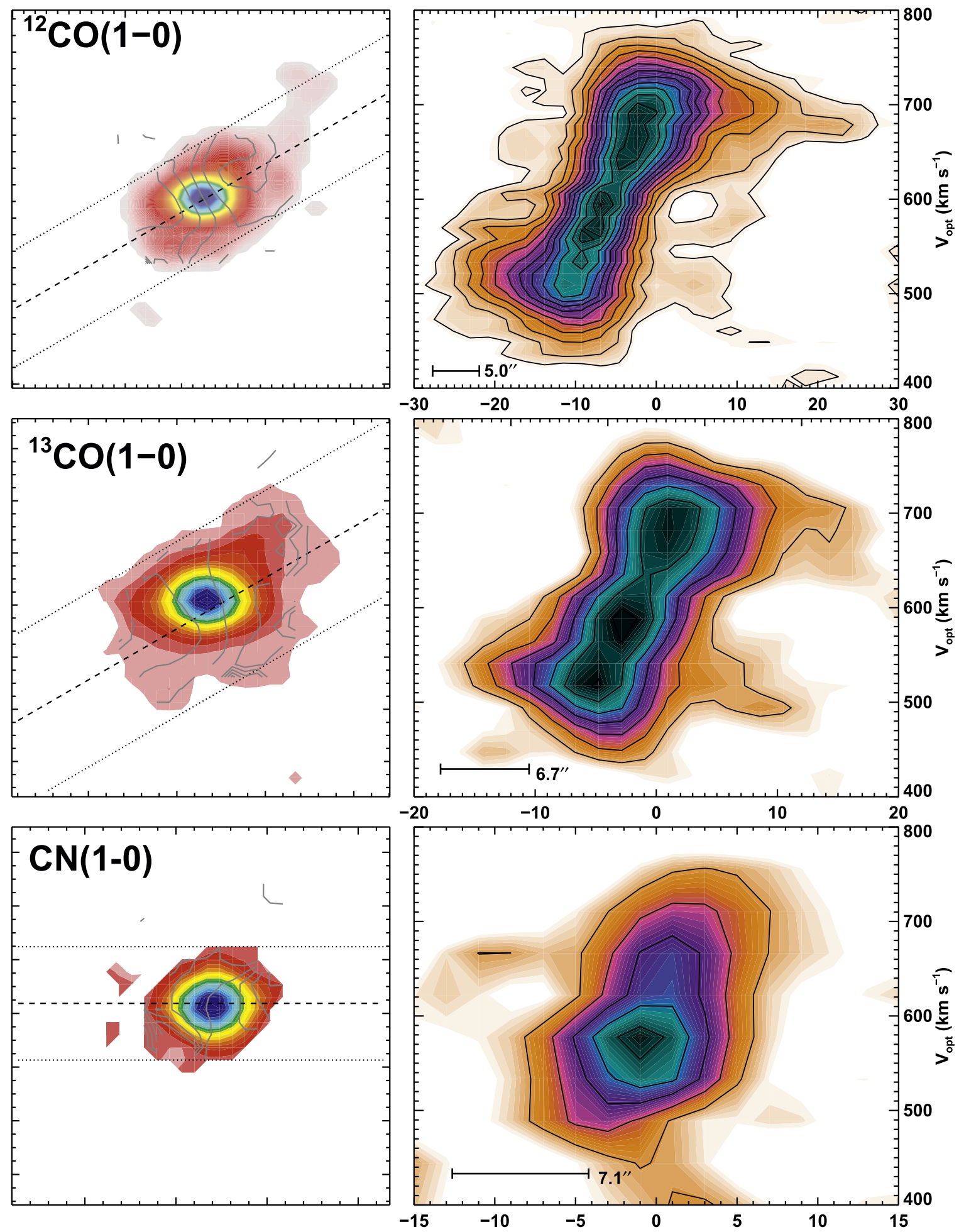

Figure 9. PV diagrams are shown for ${ }^{12} \mathrm{CO}(1-0)$ (top), ${ }^{13} \mathrm{CO}(1-0)$ (middle), and $\mathrm{CN}(1-0)$ (bottom). The left-hand images show the moment0 map of each molecular species. The dotted line denotes the bisector at the chosen position angle across the cube to create the PV diagram (right). The dotted-dashed lines denote the upper and lower bounds for the sum for each sliced region. Both ${ }^{12} \mathrm{CO}$ and ${ }^{13} \mathrm{CO}$ share a common position angle, and show signs of regular rotation. The $\mathrm{CN}$ required a different position angle, possibly suggesting an origin distinct from the other molecular tracers. The $\mathrm{CN}$ could also be tracing a different (denser) gas phase than both ${ }^{12} \mathrm{CO}$ and ${ }^{13} \mathrm{CO}$ (see Section 3.2).

providing a nonzero contribution to the far-IR luminosity. We further investigate the SFR to determine if either of these caveats play a significant role.

\subsection{Radio-determined Star Formation}

We also evaluated the SFR indicated by the centimeter- and millimeter-wave radio continuum emission using the following 


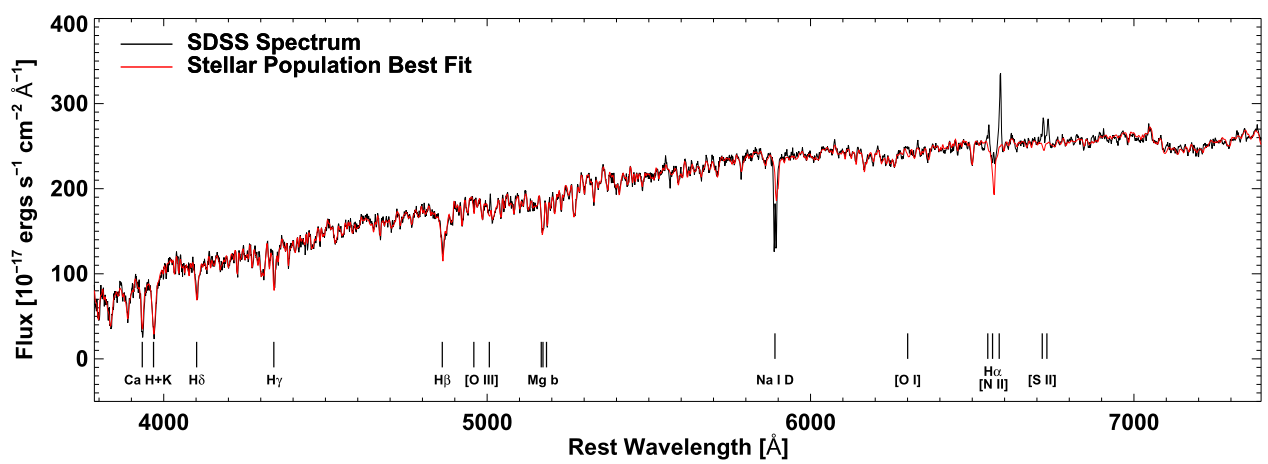

Figure 10. Nuclear spectrum of NGC 5195 (black) overlaid with the best fit combination of stellar models (red) from MILES (Vazdekis et al. 2010) and PPXF (Cappellari \& Emsellem 2004). Optical lines of note are labeled. The stellar models that best fit these data were a combination of intermediate-aged ( $1 \mathrm{Gyr})$ and older ( $\sim 10 \mathrm{Gyr})$ stellar populations (shown in Figure 11).

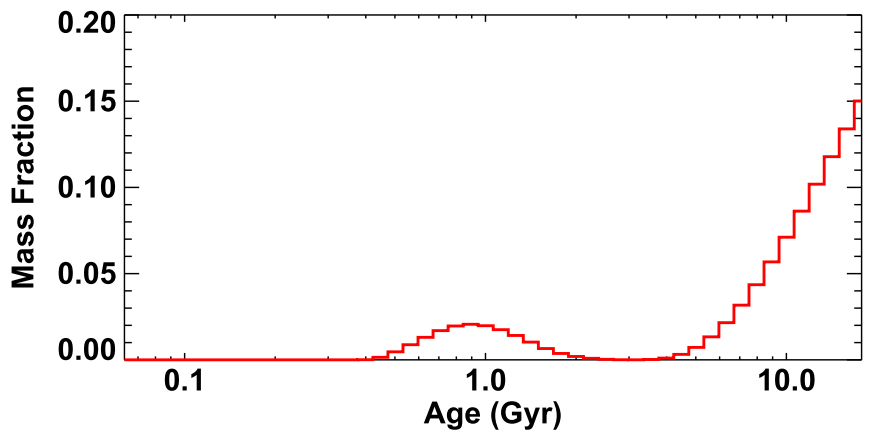

Figure 11. Probability distribution function of the stellar populations in NGC 5195 fitted to the central spectrum from SDSS DR12 (Alam et al. 2015). We used PPXF (Cappellari \& Emsellem 2004) to fit a smoothed star-formation history using the MILES library (Vazdekis et al. 2010) with a Salpeter IMF. NGC 5195 is primarily composed of older ( $\gtrsim 10 \mathrm{Gyr})$ stars, but has a significant fraction $(\approx 20 \%)$ of intermediate-aged stars $(\approx 1 \mathrm{Gyr})$.

calibration to the radio-SFR relation from Murphy et al. (2011, 2013):

$$
\begin{aligned}
& \left(\frac{\mathrm{SFR}_{\nu}}{M_{\odot} \mathrm{yr}^{-1}}\right)=10^{-27} \\
& \times\left[2.18\left(\frac{T_{\mathrm{ex}}}{10^{4} \mathrm{~K}}\right)^{0.45}\left(\frac{\nu}{\mathrm{GHz}}\right)^{-0.1}+15.1\left(\frac{\nu}{\mathrm{GHz}}\right)^{\alpha_{\mathrm{NT}}}\right] \\
& \quad \times\left(\frac{L_{\nu}}{\mathrm{erg} \mathrm{s}^{-1} \mathrm{~Hz}^{-1}}\right)
\end{aligned}
$$

with excitation temperature $T_{\mathrm{ex}}=10^{4} \mathrm{~K}$ and the nonthermal spectral index $\alpha_{\mathrm{NT}}=-0.8$. From the sensitive interferometric imaging provided by the SINGS project (Kennicutt et al. 2003; Braun et al. 2007), NGC 5195 has an integrated $1.4 \mathrm{GHz}$ flux density of $\sim 17 \mathrm{mJy}$ within the ${ }^{12} \mathrm{CO}$-detected region. This corresponds to a $1.4 \mathrm{GHz}$ SFR of $\sim 0.14 M_{\odot} \mathrm{yr}^{-1}$, slightly higher than the revised Lanz et al. (2013) integrated SFR. We, therefore, conclude that the centimeter-wave radio emission associated with NGC 5195 is predominantly produced by SF, rather than radio-loud AGN activity. This suggests that, if the nucleus of NGC 5195 is indeed currently active (as the molecular lines seem to suggest), it may be operating in the so-called radio-quiet or "high-excitation" mode characteristic of efficiently accreting massive black holes (Heckman \& Best 2014), and consistent with the weak X-rays reported by Schlegel et al. (2016). Alternatively, AGN signatures at optical and X-ray wavelengths previously reported by Moustakas et al. (2010) and Terashima \& Wilson (2004), respectively, may in fact be related to stellar processes or shocks occurring in the vicinity of the NGC 5195 nucleus (Lisenfeld \& Völk 2010). If such a scenario in which the massive black hole in the center of NGC 5195 is in a quiescent state is indeed true, it is not clear what mechanism is responsible for halting AGN fueling given the abundant availability of cold gas. Additional detailed studies of the gas kinematics in the ambient environment of the NGC 5195 nucleus will be needed to disentangle these possibilities in the future.

At the level of the integrated flux density of $\sim 1 \mathrm{mJy}$ of the compact $106 \mathrm{GHz}$ emission, the SFR predicted by Equation (5) is $\sim 0.07 M_{\odot} \mathrm{yr}^{-1}$. This is a lower limit, as it is likely that CARMA resolved out the diffuse, lower-level emission (seen in the $1.4 \mathrm{GHz}$ map in Braun et al. 2007). Even with this caveat, the predicted SFR based on the millimeter continuum emission is similarly consistent with a pure SF origin.

\subsection{Resolved Star Formation}

In order to correctly differentiate star formation taking place in the molecular gas in NGC 5195, we use the reduced $70 \mu \mathrm{m}$ image (see: Section 2.1) from Herschel PACS (Poglitsch et al. 2010). The map was then converted to a $\Sigma_{\text {SFR }}$ map using Equation (21) from Calzetti et al. (2010). We were able to directly compare this map to the $\Sigma_{\mathrm{H}_{2}}$ map, which we derived from the ${ }^{12} \mathrm{CO}(1-0)$ using Equation (3), after converting the unclipped integrated intensity map using the Kelvin per Jansky factor for the CARMA map (listed in Table 2). We then regridded the $70 \mu \mathrm{m}$ map to match the ${ }^{12} \mathrm{CO}$ map using the IDL task hastrom. ${ }^{20}$ We used the clipped moment maps (Figure 7) to define the comparison region (which contains "real" emission far above the noise of the map, with a total area of $3.3 \mathrm{kpc}^{2}$ ).

We first calculated the total SFR by summing the $\Sigma_{\text {SFR }}$ corresponding to ${ }^{12} \mathrm{CO}$ emission, $\mathrm{SFR}_{\text {tot }}=0.072 M_{\odot} \mathrm{yr}^{-1}$, that is a factor of 1.6 smaller than the revised SFR in Section 4.2, and consistent with the $106 \mathrm{GHz}$ free-free estimate for the SFR. This is possibly due to the smaller area subtended by the detected ${ }^{12} \mathrm{CO}$ emission. We then calculated the resolved depletion time for NGC 5195 by dividing the matched $\Sigma_{\mathrm{H}_{2}}$ by $\Sigma_{\mathrm{SFR}}$, shown in Figure 12. The average depletion timescale

\footnotetext{
${ }^{20}$ http://idlastro.gsfc.nasa.gov/ftp/pro/astrom/hastrom.pro
} 


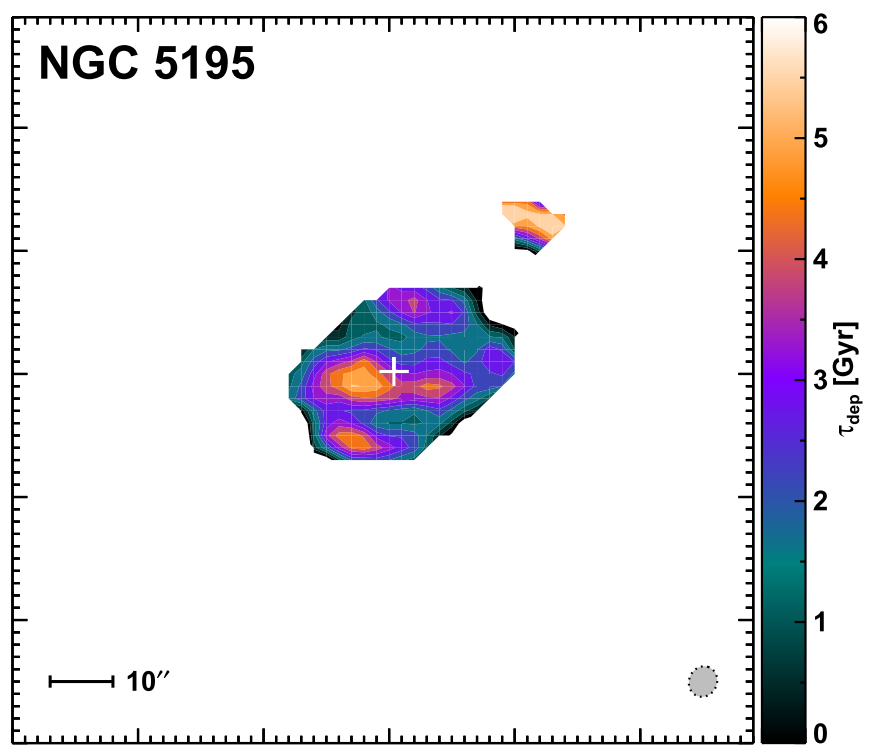

Figure 12. Depletion time map for gas in NGC 5195. The depletion time was calculated by dividing the matched $\Sigma_{\mathrm{H}_{2}}$ by $\Sigma_{\mathrm{SFR}}$. The CARMA beam is shown in the bottom right (gray). The mean depletion time for the gas in NGC 5195 is $\left\langle\tau_{\text {dep }}\right\rangle=3.08$ Gyr. The position of the radio peak is shown as a white cross, which is not spatially coincident with the highest $\tau_{\text {dep }}$, but is in its vicinity.

(taken only from unmasked pixels with positive values) is 3.08 Gyr (with a range between 1 and $6 \mathrm{Gyr}$ ).

When we include helium, this depletion time increases to $4.31 \mathrm{Gyr}$. This depletion timescale is a factor of $\approx 2$ larger than that found in normal star-forming galaxies (Bigiel et al. 2011; Saintonge et al. 2011; Leroy et al. 2013). On the other hand, this depletion time is more consistent with the molecular gas depletion time found for ATLAS ${ }^{3 \mathrm{D}}$ early-type galaxies (Davis et al. 2014). Given that NGC 5195 is an early-type galaxy, the depletion timescale consistent with early-type galaxies is fitting.

Overall, despite having taken part in the interactions with M51a a few hundred Myr ago, the gas in NGC 5195 appears to have resettled and returned to forming stars at normal efficiency (for an early-type galaxy). This provides an update to the observations of Kohno et al. (2002), who suggested that the molecular disk was gravitationally stable against collapse, suppressing star formation in this system. Our data suggest that NGC 5195 has returned to forming stars normally for its type (and its molecular gas content), which might include shear forces capable of reducing the star-formation efficiency by $\sim 2$ (Martig et al. 2013; Davis et al. 2014), consistent with other early-type galaxies.

The level of star formation we currently observe in NGC 5195 is consistent with a star-formation history that includes an enhancement in star formation corresponding to a near approach and subsequent interaction with M51a (possibly including a super-efficient burst of star formation) that has declined for the past $1 \mathrm{Gyr}$, followed by a resettling of the molecular disk and the re-establishment of efficient, normalmode star formation consistent with its early-type classification.

\section{CONCLUSIONS}

We used CARMA to map four molecular gas tracers in NGC 5195, detecting ${ }^{12} \mathrm{CO}(1-0),{ }^{13} \mathrm{CO}(1-0), \mathrm{CN}(1-0)$ and
$\mathrm{CS}(2-1)$, and measured the $106 \mathrm{GHz}$ continuum emission in this galaxy, in addition to detections of $\mathrm{HCN}(1-0)$ and $\mathrm{HCO}^{+}(1-0)$ with the $O S O 20 \mathrm{~m}$. We also fitted the SDSS DR12 nuclear spectrum of NGC 5195 with stellar population models using a smoothed star-formation history.

1. We find that our detections of ${ }^{12} \mathrm{CO}(1-0),{ }^{13} \mathrm{CO}(1-0)$ and $\mathrm{HCN}(1-0)$ are consistent with previous observations and we provide the first measurements of $\mathrm{CN}(1-0), \mathrm{CS}(2-1)$, $\mathrm{HCO}^{+}(1-0)$ and $106 \mathrm{GHz}$ continuum for NGC 5195 .

2. $\mathcal{R}_{12 / 13}$ has been updated for NGC 5195 to be $11.4 \pm 0.5$, which is much more consistent with a settled molecular distribution in a typical star-forming galaxy than a ULIRG, which tend to have large $\mathcal{R}_{12 / 13}$ (due to their disrupted molecular gas distributions).

3. The $\mathrm{CN}(1-0)$ emission found in the center of the galaxy appears to have kinematics that are different from the other molecular gas tracers studied. We suggest that the $\mathrm{CN}$ is tracing a diffuse component of the molecular gas that is found along the $x 2$ orbits perpendicular to the stellar bar.

4. The molecular line ratios suggest that the dense gas $\left(n \gtrsim 10^{5} \mathrm{~cm}^{-3}\right)$ fraction is low.

5. The stellar population fit to the nuclear spectrum of NGC 5195 contains an $80 \%$ mass fraction of old ( $\gtrsim 10$ Gyr) stars and a $20 \%$ mass fraction of intermediate-aged $(\approx 1 \mathrm{Gyr})$ stars, consistent (within uncertainties) with an enhancement in star formation taking place starting during first approach and through the recent interaction with M51a.

6. The centimeter and millimeter continuum observations provide evidence that NGC 5195 does not contain a buried AGN (or the AGN must be weak both in X-rays and very radio quiet). The SFRs determined using the radio emission also support the claim that the molecular gas in NGC 5195 is forming stars at normal efficiency.

7. The resolved star-formation relation taken from the Herschel $70 \mu \mathrm{m}$ maps of NGC 5195 is consistent with the molecular gas-forming stars at the efficiency observed for early-type galaxies.

Although NGC 5195 has undergone a substantial interaction with M51a in the recent past $(\sim 1 / 2 \mathrm{Gyr})$, it appears in the intervening time that its molecular gas has resettled into a disk and re-established efficient star formation for its morphological type.

The authors thank Dr Henrik Olofsson for carrying out the observations with the $20 \mathrm{~m}$ telescope for them. KA thanks the anonymous referee for an insightful report that has markedly improved the manuscript, and M. Daprà for GILDAS advice. Support for KA is provided by NASA through the Hubble Fellowship Grant \#HST-HF2-51352.001 awarded by the Space Telescope Science Institute, which is operated by the Association of Universities for Research in Astronomy, Inc., for NASA, under contract NAS5-26555. SA acknowledges support from the Swedish National Science Council Grant 6212011-4143. LL acknowledges support for this work provided by NASA through an award issued by JPL/Caltech. KN acknowledges support from NASA through the Spitzer Space Telescope.

Support for CARMA construction was derived from the Gordon and Betty Moore Foundation, the Kenneth T. and Eileen L. Norris Foundation, the James S. McDonnell 
Foundation, the Associates of the California Institute of Technology, the University of Chicago, the states of California, Illinois, and Maryland, and the National Science Foundation. Ongoing CARMA development and operations are supported by the National Science Foundation under a cooperative agreement, and by the CARMA partner universities. The $20 \mathrm{~m}$ telescope is operated by Onsala Space Observatory (OSO), the Swedish National Facility for Radio Astronomy.

The National Radio Astronomy Observatory is a facility of the National Science Foundation operated under cooperative agreement by Associated Universities, Inc. This research has made use of the NASA/IPAC Extragalactic Database (NED) which is operated by the Jet Propulsion Laboratory, California Institute of Technology, under contract with the National Aeronautics and Space Administration. This work is based [in part] on observations made with the Spitzer Space Telescope, which is operated by the Jet Propulsion Laboratory, California Institute of Technology under a contract with NASA. Herschel is an ESA space observatory with science instruments provided by European-led Principal Investigator consortia and with important participation from NASA. Funding for the Sloan Digital Sky Survey IV has been provided by the Alfred P. Sloan Foundation, the US Department of Energy Office of Science, and the Participating Institutions. SDSS-IV acknowledges support and resources from the Center for HighPerformance Computing at the University of Utah. The SDSS web site is www.sdss.org.

Facilities: CARMA, Herschel, OSO: 20m, SDSS, Spitzer.

\section{REFERENCES}

Aalto, S., Booth, R. S., Black, J. H., \& Johansson, L. E. B. 1995, A\&A, 300,369

Aalto, S., Garcia-Burillo, S., Muller, S., et al. 2015, A\&A, 574, A85

Aalto, S., \& Rydbeck, G. 2001, in ASP Conf. Ser. 249, The Central Kiloparsec of Starbursts and AGN: The La Palma Connection, ed. J. H. Knapen et al. (San Francisco, CA: ASP), 719

Aladro, R., Viti, S., Bayet, E., et al. 2013, A\&A, 549, A39

Alam, S., Albareti, F. D., Allende Prieto, C., et al. 2015, ApJS, 219, 12

Alatalo, K., Appleton, P. N., Lisenfeld, U., et al. 2015a, ApJ, 812, 117

Alatalo, K., Blitz, L., Young, L. M., et al. 2011, ApJ, 735, 88

Alatalo, K., Cales, S. L., Appleton, P. N., et al. 2014, ApJL, 794, L13

Alatalo, K., Cales, S. L., Rich, J. A., et al. 2016a, ApJS, 224, 38

Alatalo, K., Crocker, A. F., Aalto, S., et al. 2015b, MNRAS, 450, 3874

Alatalo, K., Davis, T. A., Bureau, M., et al. 2013, MNRAS, 432, 1796

Alatalo, K., Lisenfeld, U., Lanz, L., et al. 2016b, ApJ, 827, 106

Alloin, D. (ed.) 2006, Physics of Active Galactic Nuclei at all Scales, Vol. 693 (Berlin: Springer)

Appleton, P. N., Mundell, C., Bitsakis, T., et al. 2014, ApJ, 797, 117

Baade, W. 1958, RA, 5, 3

Baldry, I. K., Glazebrook, K., Brinkmann, J., et al. 2004, ApJ, 600, 681

Bell, E. F., McIntosh, D. H., Katz, N., \& Weinberg, M. D. 2003, ApJS, 149,289

Bigiel, F., Leroy, A., Walter, F., et al. 2008, AJ, 136, 2846

Bigiel, F., Leroy, A. K., Walter, F., et al. 2011, ApJL, 730, L13

Blake, G. A., Masson, C. R., Phillips, T. G., \& Sutton, E. C. 1986, ApJS, 60, 357

Bock, D. C.-J., Bolatto, A. D., Hawkins, D. W., et al. 2006, Proc. SPIE, 6267, 626713

Bolatto, A. D., Wolfire, M., \& Leroy, A. K. 2013, ARA\&A, 51, 207

Boulade, O., Sauvage, M., Altieri, B., et al. 1996, A\&A, 315, L85

Braun, R., Oosterloo, T. A., Morganti, R., Klein, U., \& Beck, R. 2007, A\&A, 461,455

Calzetti, D., Wu, S.-Y., Hong, S., et al. 2010, ApJ, 714, 1256

Cappellari, M., \& Emsellem, E. 2004, PASP, 116, 138

Cluver, M. E., Appleton, P. N., Ogle, P., et al. 2013, ApJ, 765, 93

da Cunha, E., Charlot, S., \& Elbaz, D. 2008, MNRAS, 388, 1595

Dale, D. A., Cohen, S. A., Johnson, L. C., et al. 2009, ApJ, 703, 517

Davis, T. A., Alatalo, K., Sarzi, M., et al. 2011, MNRAS, 417, 882
Davis, T. A., Young, L. M., Crocker, A. F., et al. 2014, MNRAS, 444, 3427 de Vaucouleurs, G., de Vaucouleurs, A., Corwin, H. G., Jr., et al. 1991, Third Reference Catalogue of Bright Galaxies, Vol. 1, 2, 3 (New York, NY: Springer)

Dobbs, C. L., Theis, C., Pringle, J. E., \& Bate, M. R. 2010, MNRAS, 403, 625 Donovan Meyer, J., Koda, J., Momose, R., et al. 2013, ApJ, 772, 107

Dressler, A., \& Gunn, J. E. 1983, ApJ, 270, 7

Faber, S. M., Willmer, C. N. A., Wolf, C., et al. 2007, ApJ, 665, 265

Fazio, G. G., Hora, J. L., Allen, L. E., et al. 2004, ApJS, 154, 10

French, K. D., Yang, Y., Zabludoff, A., et al. 2015, ApJ, 801, 1

Ginard, D., Fuente, A., García-Burillo, S., et al. 2015, A\&A, 578, A49

Greenawalt, B., Walterbos, R. A. M., Thilker, D., \& Hoopes, C. G. 1998, ApJ, 506,135

Heckman, T. M., \& Best, P. N. 2014, ARA\&A, 52, 589

Helou, G., Roussel, H., Appleton, P., et al. 2004, ApJS, 154, 253

Hickson, P. 1982, ApJ, 255, 382

Ho, L. C., Filippenko, A. V., \& Sargent, W. L. W. 1997, ApJS, 112, 315 Holmberg, E. 1958, MeLuS, 136, 1

Hopkins, P. F., Hernquist, L., Cox, T. J., et al. 2006, ApJS, 163, 1

Hopkins, P. F., Hernquist, L., Cox, T. J., \& Kereš, D. 2008, ApJS, 175, 356

Johnson, K. E., Hibbard, J. E., Gallagher, S. C., et al. 2007, AJ, 134, 1522

Kennicutt, R. C., Jr. 1998, ApJ, 498, 541

Kennicutt, R. C., Jr., Armus, L., Bendo, G., et al. 2003, PASP, 115, 928

Kennicutt, R. C., \& Evans, N. J. 2012, ARA\&A, 50, 531

Kewley, L. J., Groves, B., Kauffmann, G., \& Heckman, T. 2006, MNRAS, 372,961

Ko, J., Hwang, H. S., Lee, J. C., \& Sohn, Y.-J. 2013, ApJ, 767, 90

Kohno, K., Tosaki, T., Matsushita, S., et al. 2002, PASJ, 54, 541

Lacy, M., Storrie-Lombardi, L. J., Sajina, A., et al. 2004, ApJS, 154, 166

Lanz, L., Zezas, A., Brassington, N., et al. 2013, ApJ, 768, 90

Lepp, S., \& Dalgarno, A. 1996, A\&A, 306, L21

Leroy, A. K., Walter, F., Brinks, E., et al. 2008, AJ, 136, 2782

Leroy, A. K., Walter, F., Sandstrom, K., et al. 2013, AJ, 146, 19

Lisenfeld, U., Appleton, P. N., Cluver, M. E., et al. 2014, A\&A, 570, A24

Lisenfeld, U., \& Völk, H. J. 2010, A\&A, 524, A27

Martig, M., Crocker, A. F., Bournaud, F., et al. 2013, MNRAS, 432, 1914

Martín, S., Kohno, K., Izumi, T., et al. 2015, A\&A, 573, A116

Matsushita, S., Kawabe, R., Kohno, K., Tosaki, T., \& Vila-Vilaró, B. 2010, PASJ, 62, 409

McMullin, J. P., Waters, B., Schiebel, D., Young, W., \& Golap, K. 2007, in ASP Conf. Ser. 376, Astronomical Data Analysis Software and Systems XVI, ed. R. A. Shaw, F. Hill, \& D. J. Bell (San Francisco, CA: ASP), 127 Mentuch Cooper, E., Wilson, C. D., Foyle, K., et al. 2012, ApJ, 755, 165

Moreno, J., Torrey, P., Ellison, S. L., et al. 2015, MNRAS, 448, 1107 Moustakas, J., Kennicutt, R. C., Jr., Tremonti, C. A., et al. 2010, ApJS, 190, 233 Murphy, E. J., Condon, J. J., Schinnerer, E., et al. 2011, ApJ, 737, 67 Murphy, E. J., Stierwalt, S., Armus, L., Condon, J. J., \& Evans, A. S. 2013, ApJ, 768, 2

Parkin, T. J., Wilson, C. D., Schirm, M. R. P., et al. 2013, ApJ, 776, 65 Poglitsch, A., Waelkens, C., Geis, N., et al. 2010, A\&A, 518, L2

Quintero, A. D., Hogg, D. W., Blanton, M. R., et al. 2004, ApJ, 602, 190 Rodriguez-Franco, A., Martin-Pintado, J., \& Fuente, A. 1998, A\&A, 329, 1097 Roussel, H. 2013, PASP, 125, 1126

Roussel, H., Helou, G., Hollenbach, D. J., et al. 2007, ApJ, 669, 959

Rowlands, K., Wild, V., Nesvadba, N., et al. 2015, MNRAS, 448, 258

Sage, L. J. 1990, A\&A, 239, 125

Saintonge, A., Kauffmann, G., Wang, J., et al. 2011, MNRAS, 415, 61

Salo, H., \& Laurikainen, E. 2000, MNRAS, 319, 377

Salpeter, E. E. 1955, ApJ, 121, 161

Sault, R. J., Teuben, P. J., \& Wright, M. C. H. 1995, in ASP Conf. Ser. 77, Astronomical Data Analysis Software and Systems IV, ed. R. A. Shaw,

H. E. Payne, \& J. J. E. Hayes (San Francisco, CA: ASP), 433

Schawinski, K., Urry, C. M., Simmons, B. D., et al. 2014, MNRAS, 440, 889 Schlegel, E. M., Jones, C., Machacek, M., \& Vega, L. D. 2016, ApJ, 823, 75 Schmidt, M. 1959, ApJ, 129, 243

Schweizer, F. 1977, ApJ, 211, 324

Scudder, J. M., Ellison, S. L., Torrey, P., Patton, D. R., \& Mendel, J. T. 2012, MNRAS, 426, 549

Skrutskie, M. F., Cutri, R. M., Stiening, R., et al. 2006, AJ, 131, 1163 Smith, J. 1982, ApJ, 261, 463

Solomon, P. M., \& Vanden Bout, P. A. 2005, ARA\&A, 43, 677

Spillar, E. J., Oh, S. P., Johnson, P. E., \& Wenz, M. 1992, AJ, 103, 793

Strateva, I., Ivezić, Ž., Knapp, G. R., et al. 2001, AJ, 122, 1861

Terashima, Y., \& Wilson, A. S. 2004, ApJ, 601, 735

Tikhonov, N. A., Galazutdinova, O. A., \& Tikhonov, E. N. 2009, AstL, 35,599 
Tinsley, B. M. 1978, ApJ, 222, 14

Ueda, J., Iono, D., Yun, M. S., et al. 2014, ApJS, 214, 1

van der Tak, F. F. S., Black, J. H., Schöier, F. L., Jansen, D. J., \& van Dishoeck, E. F 2007, A\&A, 468, 627

Vazdekis, A., Sánchez-Blázquez, P., Falcón-Barroso, J., et al. 2010, MNRAS, 404, 1639
Walker, L. M., Johnson, K. E., Gallagher, S. C., et al. 2010, AJ, 140, 1254

Werner, M. W., Roellig, T. L., Low, F. J., et al. 2004, ApJS, 154, 1

Yesuf, H. M., Faber, S. M., Trump, J. R., et al. 2014, ApJ, 792, 84

Young, L. M., Bureau, M., Davis, T. A., et al. 2011, MNRAS, 414, 940

Young, L. M., Scott, N., Serra, P., et al. 2014, MNRAS, 444, 3408

Zabludoff, A. I., Zaritsky, D., Lin, H., et al. 1996, ApJ, 466, 104 